\title{
Prediction of beam losses during crab cavity quenches at the high luminosity LHC
}

\author{
R. Apsimon, ${ }^{*}$ G. Burt, A. Dexter, and N. Shipman \\ Engineering Department, Lancaster University, LA1 4YW, United Kingdom and Cockcroft Institute, \\ Daresbury Laboratory, Warrington WA4 4AD, United Kingdom
}

\author{
A. Castilla, A. Macpherson, K. Ness Sjobak, A. Santamaria Garcia, and N. Stapley \\ CERN, Geneva 1211, Switzerland \\ A. Alekou ${ }^{\dagger}$ and R. B. Appleby \\ School of Physics and Astronomy, The University of Manchester, M13 9PL, United Kingdom \\ and Cockcroft Institute, Daresbury Laboratory, Warrington WA4 4AD, United Kingdom
}

(Received 4 December 2018; published 18 June 2019)

\begin{abstract}
Studies of the crab cavities at KEKB revealed that the rf phase could shift by up to $50^{\circ}$ within $\sim 50 \mu \mathrm{s}$ during a quench; while the cavity voltage is still at approximately $75 \%$ of its nominal amplitude. If such a failure were to occur on the HL-LHC crab cavities, it is likely that the machine would sustain substantial damage to the beam line and surrounding infrastructure due to uncontrolled beam loss before the machine protection system could dump the beam. We have developed a low-level rf system model, including detuning mechanisms and beam loading, and use this to simulate the behavior of a crab cavity during a quench, modeling the low-level rf system, detuning mechanisms and beam loading. We supplement this with measurement data of the actual rf response of the proof of principle double-quarter wave crab cravity during a quench. Extrapolating these measurements to the HL-LHC, we show that Lorentz force detuning is the dominant effect leading to phase shifts in the crab cavity during quenches; rather than pressure detuning which is expected to be dominant for the KEKB crab cavities. The total frequency shift for the HL-LHC crab cavities during quenches is expected to be about $460 \mathrm{~Hz}$, leading to a phase shift of no more than $3^{\circ}$. The results of the quench model are read into a particle tracking simulation, SixTrack, and used to determine the effect of quenches on the HL-LHC beam. The quench model has been benchmarked against the KEKB experimental measurements. In this paper we present the results of the simulations on a crab cavity failure for HL-LHC as well as for the SPS and show that beam loss is negligible when using a realistic low-level rf response.
\end{abstract}

DOI: 10.1103/PhysRevAccelBeams.22.061001

\section{INTRODUCTION}

The High Luminosity upgrade for the LHC (HL-LHC) [1] will use crab cavities to compensate for the luminosity reduction at the interaction points (IPs) due to the crossing angle of the counterrotating bunch trains. A crab cavity is a deflecting cavity, phased such that the centroid of the beam passes through the cavity on the zero-crossing; hence providing an effective rotation to the bunches. If the phase of the cavity field changes with respect to the bunches, then the bunch centroid experiences a kick, which can lead to

\footnotetext{
*r.apsimon@lancaster.ac.uk

'Also at CERN, Geneva, Switzerland.
}

Published by the American Physical Society under the terms of the Creative Commons Attribution 4.0 International license. Further distribution of this work must maintain attribution to the author(s) and the published article's title, journal citation, and DOI. particle losses. During a quench, the phase and amplitude of the cavity field can change relatively quickly, potentially leading to beam losses throughout the ring.

The response time of the machine protection system depends on many different factors, but has an upper limit of 3 turns of the LHC ring, or approximately $267 \mu$ s [2]. Beam losses which occur within $267 \mu$ s of a failure can potentially damage the LHC beam line.

Measurements of the crab cavities installed at KEKB have revealed that the rf phase shifts by up to $50^{\circ}$ within $50 \mu \mathrm{s}$ during a quench; while the cavity voltage is still approximately $75 \%$ of its nominal value [3]. Should such a failure occur on the HL-LHC crab cavities, the beam losses could cause substantial damage to the beam line. The following studies are critical as CERN could not risk installing crab cavities in HL-LHC if they have the potential to damage the machine; which would severely limit the luminosity upgrade for HL-LHC. 
In this paper, we investigate the mechanisms occurring during the quench of a crab cavity and perform a comparison between the HL-LHC crab cavities and the KEKB cavities. It should be stated that there are notable differences between the two cavities, namely the HL-LHC crab cavities operate at $2 \mathrm{~K}$, whereas for $\mathrm{KEKB}$, they operate at $4 \mathrm{~K}$. In addition to this, for KEKB, the rf system is switched off as soon as a quench is detected, while for HL-LHC, it is intended for the rf system to remain on until the beam has been dumped.

Several design studies for the HL-LHC crab cavities include the double-quarter (DQW) wave [4], rf dipole (RFD) [5,6] and the four-rod crab cavity (4RCC) [7]; although the 4RCC was not selected to be one of the final designs. Before installing the crab cavities in HL-LHC, prototypes of the DQW and RFD crab cavities will first be installed in Super Proton Synchrotron (SPS) for testing. Therefore we simulate the impact on the beam due to a crab cavity quench in the SPS in order to compare to measurements during SPS tests. The comparison of simulation and measurement will be used to verify the HL-LHC simulations ahead of the installation of the HL-LHC crab cavities.

Previous studies have imposed an arbitrary phase and amplitude response on the crab cavities to study beam losses [8]. In this paper we utilize a more realistic rf system model, including low-level rf (LLRF), pressure, Lorentz force, and resistive detuning mechanisms, as well as microphonics and beam loading in order to obtain a more realistic phase and amplitude evolution during crab cavity quenches.

\section{MODELING THE RF SYSTEM}

\section{A. Cavity model}

We shall consider an rf cavity driven by an rf source; which we shall assume to be a tetrode. We shall assume that the tetrode excites a total of $N$ modes in the cavity. In this case, the cavity voltage for the $i$ th mode is governed by the second order ordinary differential equation given in Eq. (1) [9].

$\frac{\mathrm{d}^{2} \mathbf{V}_{i}}{\mathrm{~d} t^{2}}+\frac{\omega_{i}}{Q_{0, i}} \frac{\mathrm{d} \mathbf{V}_{i}}{\mathrm{~d} t}+\frac{\omega_{i}}{Q_{e, i}} \sum_{k=1}^{N} \frac{\mathrm{d} \mathbf{V}_{k}}{\mathrm{~d} t}+\omega_{i}^{2} \mathbf{V}_{i}=\frac{2 \omega_{i}}{Q_{e, i}} \frac{\mathrm{d}}{\mathrm{d} t}\left[\mathbf{F} e^{-j \omega t}\right]$,

where $Q_{0, i}$ and $Q_{e, i}$ are the intrinsic and external quality factors for the $i$ th mode respectively, $k$ is the summation index over all modes, and $\omega_{i}$ is the resonant angular frequency of the $i$ th mode. The bold font is used to indicate that the functions are complex numbers and therefore vectors. $\mathbf{F}$ is the voltage corresponding to the input power, $\mathbf{P}_{\text {in }}$ from the tetrode, expressed as

$$
\mathbf{F}=\sqrt{2\left(\frac{R}{Q}\right) Q_{L} \mathbf{P}_{\text {in }}} .
$$

Where $Q_{L}$ is the loaded quality factor, given as $\frac{1}{Q_{L}}=$ $\frac{1}{Q_{0}}+\frac{1}{Q_{e}}$ and $(R / Q)$ is the shunt impedance divided by intrinsic Q-factor. For most cases, we can assume that only one mode is excited in the cavity. This is because the mode separation and bandwidth of the nearby modes are far away from the frequencies excited by the amplifier $[7,10]$. In this case, Eq. (1) is simplified to

$$
\frac{\mathrm{d}^{2} \mathbf{V}}{\mathrm{d} t^{2}}+\frac{\omega_{0}}{Q_{L}} \frac{\mathrm{d} \mathbf{V}}{\mathrm{d} t}+\omega_{0}^{2} \mathbf{V}=\frac{2 \omega_{0}}{Q_{e}} \frac{\mathrm{d}}{\mathrm{d} t}\left[\mathbf{F} e^{-j \omega t}\right]
$$

where the subscript $i$ has been removed as we only consider one mode and $\omega_{i}$ replaced with $\omega_{0}$ to be consistent with conventional notation. $\omega_{0}$ is the resonant frequency of the cavity and $\omega$ is the driver frequency from the rf source.

Under steady state conditions, Eq. (3) is the well-known equation of motion for the driven, damped harmonic oscillator, however, during a quench or other type of failure, this is not true. During a quench, $Q_{0}$ hence $Q_{L}$ decreases due to increased resistive losses. In addition, detuning mechanisms, such as Lorentz detuning [11], will change $\omega_{0}$ over time. Eq. (3) can be solved numerically if we have a model for $Q_{0}(t)$ and $\omega_{0}(t)$ during a quench.

Although Eq. (3) can be solved numerically, to study the performance of the LLRF system, the rf phase must be calculated with accuracy on the order of millidegrees as this is the expected stability of the LLRF system. To achieve this, a low-order numerical integrator, such as a 4th-order Runge-Kutta method (RK4), would need to evaluate the cavity voltage hundreds or thousands of times per rf cycle. Alternatively, a higher order integrator, such as RK12, would require evaluating the cavity voltage less often, but each evaluation step would require more computations. In either case, solving Eq. (3) accurately over hundreds or thousands of $\mathrm{rf}$ cycles requires substantial computing time.

In order to overcome the challenges of evaluating Eq. (3) accurately over many if cycles, we can make some approximations to derive an envelope equation. This allows us to calculate the cavity voltage once every rf cycle, while still providing the same degree of accuracy. We shall assume that the solution to Eq. (3) can be expressed as

$$
\mathbf{V}(t)=\mathbf{V}_{\text {cav }}(t) e^{-j \omega t},
$$

relative to a reference frequency $\omega$, where $\mathbf{V}_{\text {cav }}(t)$ is the complex envelope as a function of time, which gives information of the amplitude and phase of $\mathbf{V}(t)$ and is assumed to be a slowly varying complex function. By differentiating Eq. (4), we obtain

$$
\begin{aligned}
\frac{\mathrm{d} \mathbf{V}}{\mathrm{d} t} & =\left[\dot{\mathbf{V}}_{\mathrm{cav}}-j \omega \mathbf{V}_{\mathrm{cav}}\right] e^{-j \omega t} \\
\frac{\mathrm{d}^{2} \mathbf{V}}{\mathrm{d} t^{2}} & =\left[\ddot{\mathbf{V}}_{\mathrm{cav}}-2 j \omega \dot{\mathbf{V}}_{\mathrm{cav}}-\omega^{2} \mathbf{V}_{\mathrm{cav}}\right] e^{-j \omega t} .
\end{aligned}
$$

By substituting Eq. (5) into Eq. (3) and canceling the $e^{-j \omega t}$ term throughout, we obtain 


$$
\begin{aligned}
\ddot{\mathbf{V}}_{\mathrm{cav}} & +\left(\frac{\omega_{0}}{Q_{L}}-2 j \omega\right) \dot{\mathbf{V}}_{\mathrm{cav}}+\left[\left(\omega_{0}^{2}-\omega^{2}\right)-j \frac{\omega \omega_{0}}{Q_{L}}\right] \mathbf{V}_{\mathrm{cav}} \\
= & \frac{2 \omega_{0}}{Q_{e}}[\dot{\mathbf{F}}-j \omega \mathbf{F}] .
\end{aligned}
$$

As we assume that $\mathbf{V}_{\text {cav }}$ is slowly varying, we can neglect the $\ddot{\mathbf{V}}_{\text {cav }}$ term. For almost all rf cavities, $Q_{L} \gg 1$, hence by multiplying Eq. (6) by $\left(\frac{\omega_{0}}{Q_{L}}+2 j \omega\right)$ and neglecting terms dependent on $Q_{L}^{-2}$, we obtain

$$
\frac{\dot{\mathbf{V}}_{\mathrm{cav}}}{\omega_{0}}+\left[\frac{\omega_{0}^{2}+\omega^{2}}{4 Q_{L} \omega^{2}}+j \frac{\omega_{0}^{2}-\omega^{2}}{2 \omega \omega_{0}}\right] \mathbf{V}_{\mathrm{cav}}=\frac{j \dot{\mathbf{F}}+\omega \mathbf{F}}{\omega Q_{e}}
$$

It should be noted that Eq. (7) is valid for any mode, hence it can be applied for accelerating cavities as well as deflecting or crabbing cavities.

\section{B. Modeling pressure detuning}

As described in the following section, there are several detuning mechanisms that will change the resonant frequency of the cavity. Due to the high Q-factors associated with SRF cavities, they are very sensitive to small shifts in frequency, therefore it is important to take detuning mechanisms into account. Pressure detuning is the most challenging mechanism to model as it depends on many factors, such as temperature, location, and nature of the quench. In this section we will develop very simple models for pressure detuning in normal liquid helium (LHe) (at 4K) and superfluid LHe (at 2K). The following models are simplistic models aimed at helping to provide insight into the order of magnitudes of pressures we expect.

\section{Pressure detuning in normal LHe}

In order to determine the pressure spike from $\mathrm{LHe}$ boiling during a quench, we shall make some assumptions to help simplify the problem so that an analytical expression can be obtained: (i) The power dissipation is assumed to be constant during the quench as the stored energy dissipates from the structure. (ii) We will assume that the localized pressure spike can be approximated as the average pressure on the cavity distributed evenly over the surface. (iii) Gaseous helium behaves like an ideal gas. (iv) The quench propagates over the surface of the cavity at the speed of sound in Niobium $\left(v_{q} \approx 5000 \mathrm{~m} / \mathrm{s}\right)$. (v) The volume of the gaseous helium "bubble" expands as a sphere, whose radius increases at the speed of sound in LHe ( $v_{r} \approx 175 \mathrm{~m} / \mathrm{s}$ at $\left.4 \mathrm{~K}[12]\right)$.

The quench behavior will affect the pressure detuning, but we shall use values based on the measurements of a quench taken at CERN and presented in Sec. III. Namely, we shall assume that the stored energy dissipates from the cavity for a duration of $10 \mathrm{~ms}$ and that the total energy dissipated as heat is $H_{\text {diss }}=12.56 \mathrm{~J}$.
The specific latent heat of evaporation for LHe is $H_{L}=84.5 \mathrm{~J} / \mathrm{mol}=21.1 \mathrm{~J} / \mathrm{g}$. At $4 \mathrm{~K}$, LHe has a density of approximately $0.125 \mathrm{~g} / \mathrm{cm}^{3}$, therefore, we can calculate the mass of helium boiled during the quench as:

$$
m=\frac{H_{\text {diss }}}{H_{L}}=\frac{12.56}{21.1}=0.595 \mathrm{~g} .
$$

Therefore, the volume of LHe boiled during the quench is

$$
V_{\mathrm{LHe}}=\frac{H_{\text {diss }}}{\rho H_{L}}=4.76 \mathrm{~cm}^{3} .
$$

At $4 \mathrm{~K}$, gaseous helium has a volume 757 times larger than LHe at the same temperature at normal atmospheric pressure of $1013.25 \mathrm{mbar}(1 \mathrm{~atm})$. Therefore, a total of 3.6 litres of helium gas is produced at $1 \mathrm{~atm}$. As we assume that the power dissipation is constant during the quench, the helium is boiled at a constant rate of $0.36 \mathrm{~m}^{3} / \mathrm{s}$ at $1 \mathrm{~atm}$. As we assume that gaseous helium is an ideal gas and that the gas and liquid are both at $4 \mathrm{~K}$, we get

$$
P_{1} V_{1}=P_{2} V_{2} \text {. }
$$

We can use Eq. (10) to determine the volume and pressure of the gaseous helium as it boils as we will not assume that it is at atmospheric pressure. We have assumed that the gas volume will increase as a sphere whose radius increases at the speed of sound in LHe. However, as the quench spot grows much more rapidly on the cavity surface, this means that the gas volume will actually grow rapidly over the surface of the cavity, but very slowly radially out from the surface of the cavity:

$$
V_{2}=\frac{4}{3} \pi v_{r}^{3} t^{3}=\frac{4}{3} \pi v_{q}^{2} \tilde{v_{r}} t^{3} .
$$

Therefore the gas will grow radially out from the surface of the cavity at a rate

$$
\tilde{v}_{r}=\frac{v_{r}^{3}}{v_{q}^{2}}=0.214 \mathrm{~m} / \mathrm{s}
$$

Once the quench has fully covered the surface of the cavity, the gas can no longer expand over the surface and will now grow radially outwards at a speed $v_{r}$, given as

$$
V_{2}\left(t>t_{0}\right)=\frac{4}{3} \pi v_{r}^{2} t_{0}^{3}+A_{\text {cav }} v_{r}\left(t-t_{0}\right),
$$

where $t_{0}$ is the time after the start of the quench when the entire surface of the cavity becomes covered by the quench, $A_{\text {cav }}$ is the surface area of the cavity, which we shall assume to be $1 \mathrm{~m}^{2}$, therefore 


$$
t_{0}=\sqrt{\frac{A_{\mathrm{cav}}}{\pi v_{q}^{2}}}=0.113 \mathrm{~ms} .
$$

We know that the volume of gas at $1 \mathrm{~atm}$ increases as

$$
V_{1}=\frac{757 H_{\text {diss }}}{\rho H_{L}}\left(\frac{t}{t_{q}}\right)=0.36 t\left[\mathrm{~m}^{3}\right] \text {. }
$$

where $t_{q}=10 \mathrm{~ms}$ is the duration of the power dissipation from the cavity. We shall assume that the effect of the localized pressure spike is equal to the average pressure applied uniformly over the surface of the cavity, therefore, while the ares of the quench spot $\left(A_{\text {quench }}\right)$ is growing, the average pressure is

$\langle P\rangle=P_{2} \frac{A_{\text {quench }}}{A_{\text {cav }}}=P_{1} \frac{V_{1}}{V_{2}} \frac{\pi v_{q}^{2} t^{2}}{A_{\text {cav }}}=0.218 \frac{H_{\text {diss }} v_{q}^{2}}{v_{r}^{3} t_{q}}$.

Note that while the quench spot is growing, the average pressure is constant and using the assumed parameters, the average pressure is 1278 mbar, therefore during a quench, we expect the pressure on the cavity to be of the order of $10^{2}-10^{3}$ mbar.

Once the quench spot has covered the full surface of the cavity, the volume grows radially outwards at a speed $v_{r}=$ $175 \mathrm{~m} / \mathrm{s}$ and the average pressure on the cavity surface evolves as

$$
\langle P\rangle \approx 0.284 \frac{H_{\text {diss }}}{A_{\text {cav }} v_{r} t_{q}}\left(\frac{t}{t-t_{0}}\right) .
$$

When $t>t_{0}$, the pressure drops rapidly to an equilibrium pressure of approximately 2 mbar.

\section{Pressure detuning in superfluid LHe}

The model for pressure detuning in superfluid LHe is more straightforward than in a viscous fluid because the absence of viscosity in a superfluid means that the gaseous helium bubble can be thought of as expanding into a vacuum. We will apply many of the same assumptions as for the previous model, namely that the power dissipation as the stored energy dissipates as heat and that the gaseous helium can be treated as an ideal gas.

In addition, we will assume that the gas expands into the vacuum region of the cryostat, which has a volume, $V_{\text {cryo }} \approx 1.6 \mathrm{~m}^{3}$. The actual volume of the cryostat used for HL-LHC is $4 \mathrm{~m}^{3}$, but only about $40 \%$ of the volume is vacuum. Using the equation for an ideal gas again, we get that

$$
\langle P\rangle=P_{1} \frac{V_{1}}{V_{2}}=0.767 \frac{H_{\text {diss }}}{\rho H_{L} V_{\text {cryo }}}\left(\frac{t}{t_{q}}\right) .
$$

Therefore the pressure grows linearly during the quench up to a maximum of approximately 2.3 mbar.

Figure 1 shows a comparison of $\langle P\rangle$ vs time during the quench for normal LHe at $4 \mathrm{~K}$ (blue) and superfluid LHe at

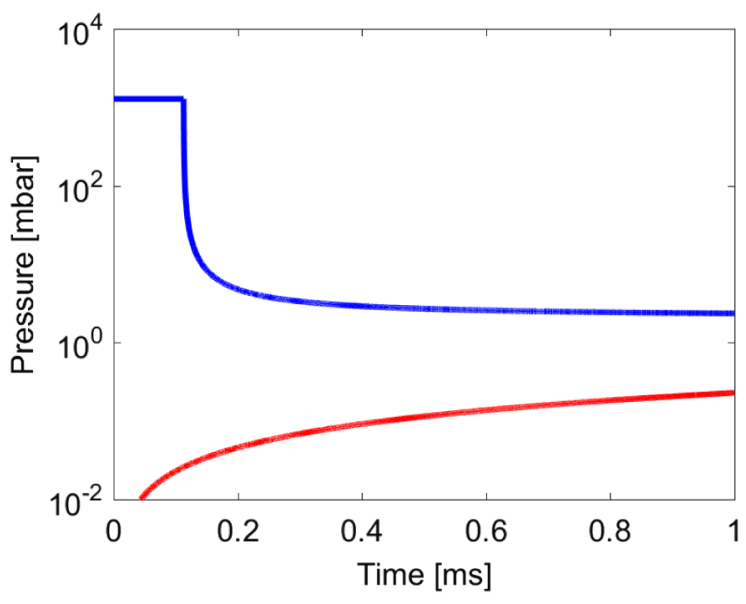

FIG. 1. Comparison of pressure vs time for gaseous helium produced by boiling LHe during a quench, showing normal LHe at $4 \mathrm{~K}$ (blue) and superfluid LHe at $2 \mathrm{~K}$ (red).

$2 \mathrm{~K}$ (red). The pressure is plotted on a logarithmic scale show how pressure varies over several orders of magnitude.

As these models are intended to provide an understanding of the order of magnitude of the pressure during a quench and that the pressure will depend on many variables, we conclude that the pressure due to boiling LHe at $4 \mathrm{~K}$ will be of the order of $10^{2}-10^{3}$ mbar and for superfluid $\mathrm{LHe}$ at $2 \mathrm{~K}$, the pressure will be of the order of $10^{-1}-10^{2}$ mbar.

\section{Frequency detuning mechanisms}

In order to determine the cavity voltage from Eq. (7), we first need to understand how to model the coefficients and right-hand side (driving terms). For now, we will neglect any feedback and control on the rf source, so the incoming rf frequency, $\omega$, will be assumed constant, as well as $Q_{e}$. The resonant frequency of the cavity, $\omega_{0}$, is affected by the cavity detuning mechanism, $Q_{L}$ depends on the behavior of the quench and the material properties and $\mathbf{F}$ on the LLRF system.

n a superconducting rf (SRF), from Eq. (7), the high loaded Q-factor $\left(\gtrsim 10^{5}\right)$ suppresses the real part of the complex coefficient on the left hand side of the equation. Hence a small frequency change can result in a relatively large phase shift. For normal conducting rf, the lower Qfactors result in less sensitivity to frequency offsets, thus detuning is often neglected. For the simulation model, we consider four detuning mechanisms; namely resistive, Lorentz, pressure, and microphonics.

\section{Resistive detuning}

If we consider the undriven form of Eq. (3), we have

$$
\frac{\mathrm{d}^{2} \mathbf{V}}{\mathrm{d} t^{2}}+\frac{\omega_{0}}{Q_{L}} \frac{\mathrm{d} \mathbf{V}}{\mathrm{d} t}+\omega_{0}^{2} \mathbf{V}=0
$$


The general solution to this is

$$
\mathbf{V}=[A \cos \omega t+B \sin \omega t] e^{-\frac{\omega_{0} t}{2 Q_{L}}}
$$

where

$$
\omega=\omega_{0} \sqrt{1-\frac{1}{4 Q_{L}^{2}}} .
$$

Therefore the resistive frequency shift is

$$
\delta f_{R}=f-f_{0}=f_{0}\left(\sqrt{1-\frac{1}{4 Q_{L}^{2}}}-1\right) .
$$

\section{Lorentz, force detuning}

The electromagnetic fields in an rf cavity apply a radiation pressure to the surface of the cavity given as

$$
P_{L}=\frac{\mu_{0} H^{2}-\varepsilon_{0} E^{2}}{4}
$$

due to the Lorentz force [11]. The stored energy in the cavity scales with $E^{2}, H^{2}$, and $|\mathbf{V}|^{2}$. The radiation pressure deforms the cavity and therefore changes the resonant frequency. The frequency shift is linearly proportional to the radiation pressure, therefore the Lorentz force detuning can be expressed as

$$
\delta f_{L}=-K_{L}\left(|\mathbf{V}|^{2}-\left|\mathbf{V}_{\text {nominal }}\right|^{2}\right),
$$

where $K_{L}$ is the Lorentz force detuning coefficient. Typically, SRF structures are designed such that they operate at the correct frequency when they are under normal operating conditions, therefore the frequency shift is taken as the change in cavity voltage from its nominal value, $\mathbf{V}_{\text {nominal }}$. As the Lorentz force detuning depends on the cavity voltage, this implies that Eq. (7) becomes nonlinear. If we make the substitution $\omega \rightarrow \omega+\delta \omega_{L}$ in Eq. (7), we can separate the envelope equation into linear and nonlinear terms.

$$
\begin{aligned}
\frac{\dot{\mathbf{V}}_{\mathrm{cav}}}{\omega_{0}}+\left[\frac{\omega_{0}^{2}+\omega^{2}}{4 Q_{L} \omega^{2}}+j \frac{\omega_{0}^{2}-\omega^{2}}{2 \omega \omega_{0}}\right] \mathbf{V}_{\mathrm{cav}} \\
-\left[\frac{\omega_{0}^{2}}{2 Q_{L} \omega^{2}}+j \frac{\omega_{0}^{2}+\omega^{2}}{2 \omega \omega_{0}}\right] \frac{\delta \omega_{L}}{\omega} \mathbf{V}_{\mathrm{cav}}=\frac{j \dot{\mathbf{F}}+\omega \mathbf{F}}{\omega Q_{e}} .
\end{aligned}
$$

In Eq. (25), $\frac{\delta \omega_{L}}{\omega} \ll 1$ for most scenarios, therefore the nonlinear terms are much smaller than the linear terms.

\section{Pressure detuning}

Low temperature SRF structures are cooled in liquid helium (LHe). During a quench, the cavity surface becomes normal conducting and power is dissipated through Ohmic losses and converted into heat. The heat in turn boils the surrounding helium, resulting in an increased pressure exerted on the cavity.

A quench typically starts at a single point and quickly spreads over the surface of the cavity. As the normal conducting region grows, the heat produced from Ohmic losses increases rapidly and causes the LHe to violently flash-boil on the outer surface of the cavity. The volumetric expansion ratio for liquid to gaseous helium is $1: 757$; thus when the LHe boils, it results in a sudden pressure increase.

If the LHe temperature is above $2.17^{\circ} \mathrm{K}$, the liquid is a normal fluid and has a viscosity. The rapidly expanding volume of gas pushes again the viscous liquid and causes an increased pressure on the cavity wall. If the LHe temperature is below $2.17^{\circ} \mathrm{K}$, the liquid is superfluid and has no viscosity. The boiling helium experiences no resistance from the LHe and the pressure increase is caused by the increase in vacuum pressure from the gaseous helium. During a quench, pressures are expected to increase up to $10^{2}-10^{3}$ mbar as the LHe boils in a normal fluid state because of the reaction force of the rapidly expanding gas on the viscous fluid. As superfluid LHe boils, the pressure increase is substantially reduced as the gaseous helium expands into the vacuum, thus the pressure increase is expected to be $\sim 10^{-2}-10^{2}$ mbar. Measurements of frequency shift vs pressure range from $0.1-100 \mathrm{~Hz} / \mathrm{mbar}$ $[13,14]$. Therefore we expect the frequency shift due to pressure detuning during a quench to be of the order of $\mathrm{kHz}$ for $\mathrm{LHe}$ above $2.17^{\circ} \mathrm{K}$ and of the order of $10^{\circ}-10^{2} \mathrm{~Hz}$ for superfluid helium.

A physically realistic model of pressure detuning is difficult as there are many unknowns, such as the location of the quench point. These unknowns affect the transition time of the quench, the pressure distribution around the cavity and therefore the actual frequency shift due to pressure. We have assumed that the frequency shift due to pressure detuning is of the form

$$
\delta f_{P}=\frac{\Delta f_{p}}{2}\left[1+\tanh \left(\frac{t-t_{\text {quench }}}{\tau_{p}}\right)\right]
$$

where $\Delta f_{p}$ is the maximum frequency shift, $t_{\text {quench }}$ is the start time of the quench and $\tau_{p}$ is the transition time. This model is not realistic because the pressure detuning begins before the quench occurs, violating causality, but this is negligible if $\tau_{p}$ is small. In addition, given that $\tau_{p}$ is small, the pressure detuning occurs rapidly and the exact form of pressure as a function of time is not important.

If $\tau_{p}$ is comparable or greater than the LLRF latency, the LLRF system can react and compensate for the detuning, which will result in a short oscillatory response until the pressure detuning stabilizes.

\section{Microphonics and mechanical oscillations}

Microphonics is the term used to describe the periodic frequency shift due to vibrations caused by nearby 
equipment, such as the cryogenic system and cooling pumps, as well as the impulse from the two previously described mechanical detuning mechanisms. These vibrations are broadband and allow the SRF structure to oscillate at its mechanical resonant frequency $[15,16]$. Assuming that the SRF structure only has one mechanical resonant frequency, we can model the microphonic detuning as

$$
\delta f_{m}=\Delta f_{m} \sin \left(\omega_{m} t+\phi\right)
$$

where $\Delta f_{m}$ is the amplitude of the microphonic frequency shift, $\omega_{m}$ the mechanical resonant angular frequency of the SRF structure and $\phi$ and arbitrary phase shift. We can extend Eq. (27) to include additional terms of different resonant frequencies if desired.

Microphonics, pressure and Lorentz force detuning are all mechanical detuning mechanisms. Therefore we can expect all three mechanisms to excite oscillations. We can define the total frequency shift, $\delta f_{\text {tot }}$, due to all detuning mechanisms as

$$
\delta f_{\text {tot }}=\delta f_{R}+\delta f_{\text {mech }}
$$

where $\delta f_{\text {mech }}$ is the frequency shift due to the mechanical detuning mechanisms. $\delta f_{\text {mech }}$ is the solution for a driven, damped harmonic oscillator where $\delta f_{L}, \delta f_{p}$, and $\delta f_{m}$ are the driving terms. A simple model can be described as [16]

$$
\ddot{\delta f_{\text {mech }}}+2 \eta \omega_{m} \dot{\delta f_{\text {mech }}}+\omega_{m}^{2} \delta f_{\text {mech }}=\ddot{\delta f} d r
$$

where $\eta$ is the mechanical damping coefficient and $\delta f_{d r}$ is the driving term given as

$$
\delta f_{d r}=\left(\delta f_{L}+\delta f_{p}+\delta f_{m}\right) e^{-j \omega_{m}\left(t-t_{\text {quench }}\right)}
$$

It is assumed that the damping term is small and can be neglected when simulated over small timescales. The mechanical resonant frequency of the rf structure is of the order of $10^{0}-10^{4} \mathrm{~Hz}$, however the pressure detuning occurs on the scale of $\mathrm{MHz}$. We shall assume that $\delta f_{\text {mech }}$ from the pressure detuning only will have a form

$$
\delta f_{\text {mech }}=\frac{\Delta f_{p}}{2}\left(1+\tanh \left(\frac{\delta t}{\tau_{p}}\right)\right)\left(1+j e^{-j \omega_{m} \delta t}\right),
$$

where $\delta t=t-t_{\text {quench }}$. If we determine the second derivative and rearrange as well as taking the approximation $1 / \tau_{p} \gg \omega_{m}$, we obtain

$$
\begin{aligned}
& \ddot{\delta f_{\text {mech }}}+\left[\omega_{m}^{2}+\frac{2}{\tau_{p}^{2}} \operatorname{sech}^{2}\left(\frac{\delta t}{\tau_{p}}\right)\right] \delta f_{\text {mech }} \\
& =\omega_{m}^{2} \delta f_{p}+\frac{\Delta f_{p}}{\tau_{p}^{2}} \operatorname{sech}^{2}\left(\frac{\delta t}{\tau_{p}}\right)\left(1+e^{-j \omega_{m} t}\right),
\end{aligned}
$$

where we see that the resonant frequency of mechanical oscillation becomes $\omega_{m}^{2}+\frac{2}{\tau_{p}} \operatorname{sech}^{2}\left(\frac{\delta t}{\tau_{p}}\right)$. This implies that during the rapid change in helium pressure, the mechanical frequency briefly increases up to $\frac{\sqrt{2}}{\tau_{p}}$, allowing the $\mathrm{rf}$ frequency to rapidly change.

In simulation, the frequency shift due to pressure detuning is determined using Eq. (31) rather than evaluating Eq. (32) because the result is the same, but requires fewer computational steps. Similarly, microphonics are modeled as in Eq. (27). The frequency shift due to Lorentz detuning is determined by evaluating

$$
\ddot{\delta f}+\omega_{m}^{2} \delta f=\omega_{m}^{2} \delta f_{L}
$$

because the detuning depends on the cavity rf voltage and must therefore be evaluated in parallel with the rf voltage. Since Lorentz detuning occurs over much longer timescales than the rf period, an RK4 integrator is unnecessary and a forward Euler integrator is used instead. For each time step, the frequency shift due to Lorentz detuning is calculated as

$$
\begin{aligned}
& \dot{\delta f_{n}}=\dot{\delta f_{n-1}}+\omega_{m}^{2}\left(\delta f_{L}-\delta f_{n-1}\right) \delta t \\
& \delta f_{n}=\delta f_{n-1}+\dot{\delta f_{n}} \delta t .
\end{aligned}
$$

\section{Low-level rf system}

In the model of the rf cavity and the detuning mechanisms, described in the previous subsections, Lorentz detuning is the only source of nonlinearities in our modeled system. As explained in the previous subsection, the nonlinear terms from Lorentz detuning are several orders of magnitude smaller than the linear terms and can therefore be neglected. As such, there is no need to simulate a sophisticated LLRF system, capable of nonlinear corrections. Hence, the LLRF system is assumed to be a proportional-integral (PI) controller $[9,17,18]$.

The cavity rf voltage is measured at regular intervals, given by the digital sampling rate; for SPS and HL-LHC simulations this is taken to be $40 \mathrm{MHz}$. The cavity rf in-phase $(I)$ and quadrature $(Q)$ voltages are measured relative to a reference clock signal. This is effectively the real and imaginary components of the cavity rf relative to the nominal rf frequency, and therefore provides all the relevant information about the cavity rf voltage. The PI controller compares the measured $I$ and $Q$ voltages to the set values stored and calculates a correction.

The correction to the cavity rf from the PI controller consists of two terms; a proportional and an integral term. The proportional term calculates a correction based on the previous measurement of the $I$ and $Q$ voltage, and corrects for fast changes in the cavity voltage. The integral term calculates a correction based on the integral (or sum) of the $I$ and $Q$ voltage over all previous times and corrects for slower changes and analogue drifts. The PI controller response is given as 


$$
\delta \mathbf{F}=c_{p}\left(\mathbf{V}_{\mathrm{cav}}-\mathbf{V}_{0}\right)+c_{i} \sum_{i}\left(\mathbf{V}_{\mathrm{cav}}-\mathbf{V}_{0}\right)
$$

where $c_{p}$ and $c_{i}$ are the gains for the proportional and integral controllers respectively. The LLRF has a latency, which is typically much longer than the digital sampling rate; for the following simulations it is taken to be $1 \mu \mathrm{s}$.

\section{E. Beam loading}

As a charged particle bunch travels through an $\mathrm{rf}$ structure, the particles interact with the rf fields. In the case of an accelerating cavity, if the bunch is accelerated, energy has been taken from the rf cavity. The stored energy in a cavity is related to the voltage as

$$
U_{\text {stored }}=\frac{V_{\mathrm{cav}}^{2}}{\omega\left(\frac{R}{Q}\right)} .
$$

Thus, a change in stored energy will result in a change in voltage. For a deflecting or crabbing cavity, the energy gain of the particle bunch depends on the transverse position of the bunch as it passes through the cavity. The PanofskyWenzel theorem [19] relates the transverse voltage, $V_{\perp}$, to the transverse variation of the longitudinal electric field

$$
\mathbf{V}_{\perp}=-\frac{j c}{\omega} \int \nabla_{\perp} \mathbf{E}_{z} \cdot d z=-\frac{j c}{\omega} \nabla_{\perp} \mathbf{V}_{z}
$$

In these simulations, it is assumed that the cavity operates in a dipole mode [20-23] and that $\mathbf{V}_{z}(x)$ varies linearly with $x$ and $\mathbf{V}_{z}(0)=0$; where $x$ is taken to be the direction of the deflecting rf field. Therefore the transverse voltage is

$$
\mathbf{V}_{\perp}=-\frac{j c}{\omega} \frac{\mathbf{V}_{z}(x)}{x}
$$

Equation (36) is valid for both transverse and longitudinal voltages, provided the appropriate definition of $R / Q$ is used. The transverse $R / Q$ can be defined by substituting Eq. (38) into Eq. (36)

$$
\left(\frac{R}{Q}\right)_{\perp}=\frac{V_{z}^{2}(x)}{\omega\left(\frac{x \omega}{c}\right)^{2} U_{\text {stored }}} .
$$

By conservation of energy, the change in stored energy, $\delta U_{\text {stored }}=q V_{\text {beam }}$, and the energy gain from the bunch is $-q V_{\text {beam }}$ where $q$ is the bunch charge and $V_{\text {beam }}$ is the voltage seen by the beam. The negative sign for the energy gain of the bunch is because a positive voltage accelerates a negatively charged particle. Assuming the change in stored energy is small, we can determine the change in cavity voltage from Eq. (36)

$$
\delta U_{\text {stored }}=q V_{\text {beam }}=\frac{2 V_{\perp}}{\omega\left(\frac{R}{Q}\right)_{\perp}} \delta V_{\perp} .
$$

If we assume that the bunch passes through the cavity with a phase error, $\phi$, then $V_{\text {beam }}=V_{z}(x) e^{j \phi}$, where $x$ is the transverse position of the bunch centroid. Therefore we can determine the instantaneous change in cavity voltage by substituting Eq. (38) into Eq. (40) as

$$
\delta \mathbf{V}_{\perp}=\frac{q \omega}{2} e^{j \phi}\left(\frac{\omega x}{c}\right)\left(\frac{R}{Q}\right)_{\perp} .
$$

Note that the change in voltage depends on the transverse and longitudinal position of the bunch. Therefore the cavity voltage depends on the beam dynamics of the bunch, conversely, the beam dynamics depends on the state of the cavity. Hence there is a complicated interaction between the cavity and the beam. However, from initial studies described later in this article, we conclude that the LLRF system is easily able to compensate for beam loading, which allows us to neglect the effect the beam has on the cavity fields, significantly simplifying the simulations.

\section{CRAB CAVITY QUENCH MEASUREMENTS}

To verify the model of the quench dynamics and its impact on the crab cavity rf system, measurements of the rf amplitude and phase in order to determine the frequency shift during quench were taken at SM18 [24], CERN, on the double quarter-wave (DQW) proof-of-principle (PoP) crab cavity. The measurements were performed in a vertical test configuration where the cavity is immersed in a liquid helium bath operating at $2 \mathrm{~K}$, and the external $\mathrm{Q}$ is chosen to be slightly lower than the intrinsic Q-factor to minimize power requirements. In order to mitigate microphonics the measurement was run as a self-excited loop (SEL) [25] where the drive frequency is locked to the cavity frequency. This configuration is not an exact replica of the setup in HL-LHC as the drive frequency will be fixed by a master oscillator for HL-LHC, and the loaded Q factor will be orders of magnitude less, but from these measurements it is possible to reconstruct the change in cavity frequency with time in order to apply this to the LLRF system model. In addition the Lorentz force detuning and pressure stability of the bare PoP cavity, with a stiffening frame, are significantly higher than in the dressed DQW cavity due to the LHe vessel, hence the frequency shift contributions will need to be separated and scaled accordingly.

The cavity is configured with a fundamental power coupler (FPC), with an external Q slightly less than the cavity ohmic $\mathrm{Q}$ prior to the quench, and a pick-up probe set to have an external $\mathrm{Q}$ factor at least an order of magnitude above the ohmic $\mathrm{Q}$ prior to the quench. The pick-up probe allows a transmitted power to be obtained to give a direct measurement of the stored energy in the cavity, given the external $\mathrm{Q}$ is measured during the calibration of the 
experiment, and also as a feedback signal to the SEL. Power is delivered to the cavity via the FPC, and we measure the input power and any power reflected back towards the source.

\section{A. Stored energy and transverse voltage}

To measure the cavity amplitude and phase we downmix the input and output waveforms with a local oscillator operating at a frequency of around $400 \mathrm{MHz}$, set to be close to the cavity frequency prior to the quench. From the experimental measurements, we obtain the amplitudes of forward $\left(P_{F}\right)$, reflected $\left(P_{R}\right)$, and transmitted $\left(P_{T}\right)$ power, as well as the respective if phases relative to local oscillator signal $\left(\phi_{F}, \phi_{R}\right.$, and $\left.\phi_{T}\right)$. In addition, the fundamental power coupler is measured to have a Q-factor, $Q_{\text {in }}=1.2 \times 10^{9}$, and the pickup probe to measure the transmitted power has a Q-factor, $Q_{T}=1.5 \times 10^{11}$. During a quench, the cavity rf system is not in equilibrium, so from the conservation of energy, where the drive is locked to the cavity frequency, we obtain

$$
P_{F}-P_{R}-P_{T}-P_{C}-\frac{d U}{d t}=0
$$

where $P_{C}$ is the power dissipated in the cavity and $U$ is the stored energy in the cavity. When the rf system and the cavity are in equilibrium, $d U / d t=0$. From the definition of Q-factor, we can relate the stored energy, $U$, to $Q_{T}$ and $P_{T}$ as

$$
U=\frac{2 Q_{T} P_{T}}{\omega} .
$$

The stored energy in the crab cavity can be related to the transverse deflecting voltage as

$$
V_{T}^{2}=k U,
$$

where $k$ has been experimentally measured previously [4] to be $1.0234 \mathrm{MV}^{2} / \mathrm{J}$ for the DQW PoP crab cavity. Figure 2 shows the stored energy (red) in the cavity, using Eqs. (43) and the measured data.

\section{B. Intrinsic Q-factor}

The intrinsic Q-factor, $Q_{0}$, can be related to the stored energy in the cavity and the power dissipated in the cavity as $Q_{0}=\frac{\omega U}{P_{C}}$. From Eqs. (42) and (43), we can define $Q_{0}$ as

$$
Q_{0}=\frac{Q_{T} P_{T}}{P_{F}-P_{R}-P_{T}-\frac{d U}{d t}},
$$

$Q_{0}$ vs time (blue) is shown in Fig. 2. The vertical axis is logarithmic because the Q-factor drops by more than two orders of magnitude over the duration of the quench. The Q-factor begins to increase again from about $35 \mathrm{~ms}$ because

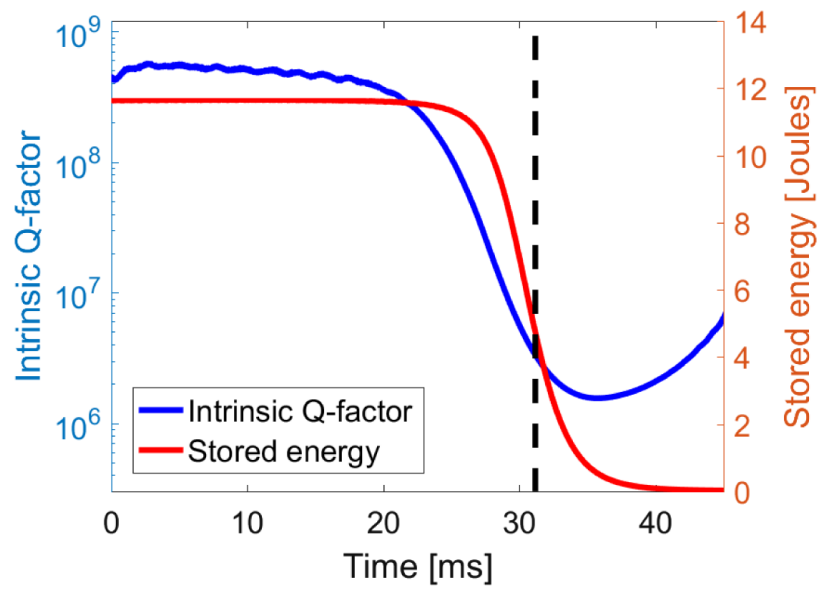

FIG. 2. Intrinsic Q-factor, $Q_{0}$, (blue) and stored energy (red) vs time in the DQW PoP crab cavity during a quench measurement at SM18, CERN. The black dashed line indicates the time of peak power dissipation, $P_{C}$.

the measured data shows a "soft" quench where the cavity recovers before the cavity becomes fully normal conducting. By comparison, in the simulations we present later in this paper, we only model "hard" quenches.

For the model of quench dynamics, we assume that the intrinsic Q-factor of the cavity decays exponentially during a quench as

$$
Q_{0}(t)=Q_{0, \mathrm{NC}}+\left(Q_{0, \mathrm{SC}}-Q_{0, \mathrm{NC}}\right) e^{-\frac{t}{\tau_{q}}},
$$

where $Q_{0, \mathrm{SC}}$ and $Q_{0, \mathrm{NC}}$ are the superconducting and normal-conducting $Q_{0}$ respectively and $\tau_{q}$ is the quench transition time. If we assume $Q_{0, \mathrm{NC}} \ll Q_{0, \mathrm{SC}}$, then we can obtain

$$
\ln \left(Q_{0}(t)\right) \approx \ln \left(Q_{0, \mathrm{SC}}-Q_{0, \mathrm{NC}}\right)-\frac{t}{\tau_{q}} .
$$

By fitting a straight line to $\ln \left(Q_{0}\right)$ vs time during the quench transition (25 $\mathrm{ms} \leq t \leq 30 \mathrm{~ms}$ in Fig. 2), we estimate the quench transition time to be $\tau_{q}=1.63 \mathrm{~ms}$.

\section{Frequency shift}

From the power amplitudes, we have been able to determine $U, V_{T}$, and $Q_{0}$ vs time. From the phase measurement, we are able to determine the frequency shift of the cavity during a quench. The frequency shift can be calculated from any of the phase data, but we have chosen to use $\phi_{T}$. Given that $\phi_{T}$ is measured in degrees, the frequency shift is given as

$$
f_{\mathrm{cav}, 0}+\delta f_{\mathrm{cav}}=\frac{1}{360} \frac{d \phi_{T}}{d t}+f_{\mathrm{LO}},
$$

where $f_{\text {cav }, 0}$ is the cavity resonant frequency before the quench, $\delta f_{\text {cav }}$ is the change in cavity frequency over time 


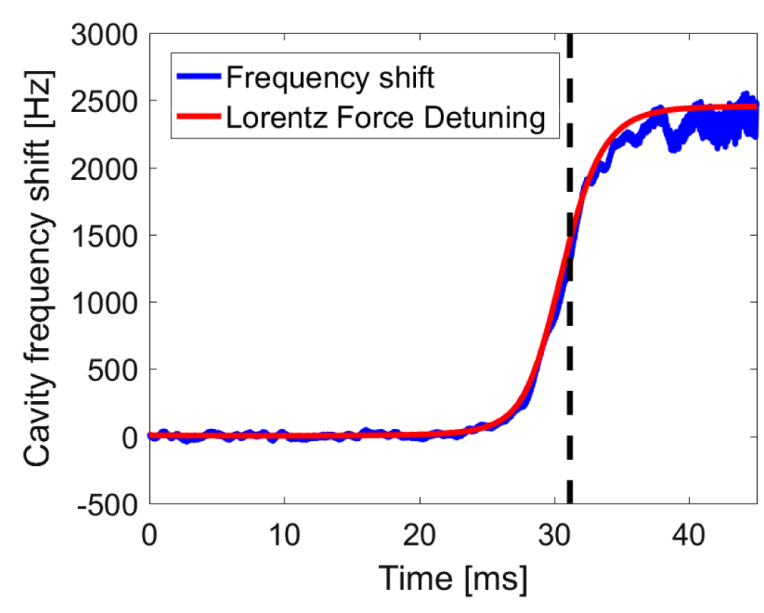

FIG. 3. Frequency shift, $\delta f_{\text {cav }}$ (blue), and calculated Lorentz force detuning (red) vs time in the DQW PoP crab cavity during a quench measurement at SM18, CERN.

and $f_{\mathrm{LO}}$ is the local oscillator frequency. Figure 3 shows the frequency shift, $\delta f_{\text {cav }}$ (blue), vs time. As can be seen the frequency shift measured is very close to the calculated detuning from the stored energy and the simulated detuning factor. This provides confidence that the rf system is indeed frequency locked to the cavity frequency. In the measurement data before the quench, the cavity resonant frequency is measured to be $3.005 \mathrm{kHz}$ higher than the local oscillator frequency; this has been subtracted from the data to only show the change in frequency during the quench. From Eq. (24), we can calculate the frequency shift due to Lorentz force detuning (red), given that the Lorentz detuning factor is measured to be $K_{L}=-206 \mathrm{~Hz} / \mathrm{MV}^{2}$ [4] for the DQW PoP crab cavity.

By plotting the frequency shift with the predicted Lorentz force detuning subtracted (Fig. 4, blue curve), we are able to observe the effect of other detuning mechanisms, such as pressure detuning as well as mechanical vibrations in the

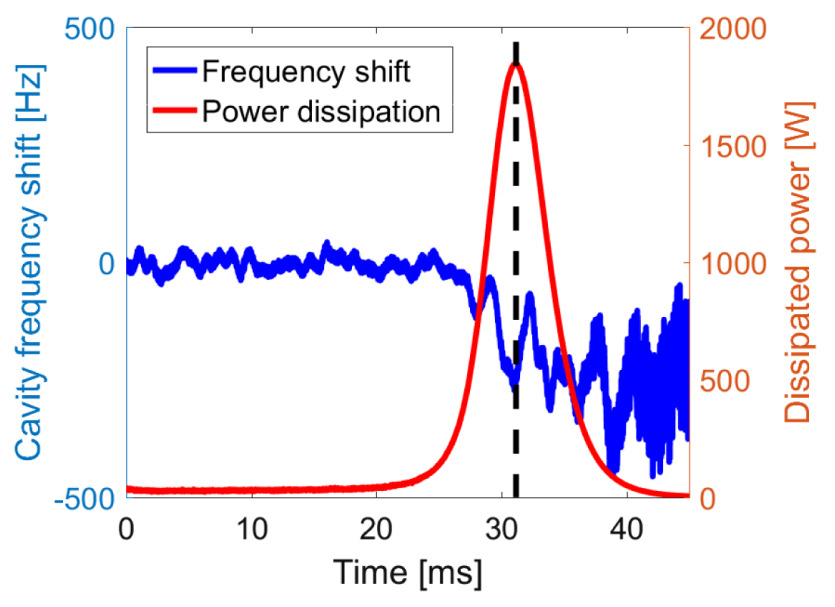

FIG. 4. Frequency shift with Lorentz force detuning subtracted (blue) and dissipated power (red) vs time in the DQW PoP crab cavity during a quench measurement at SM18, CERN. cavity at several different frequencies; although we cannot be certain that the oscillations later in the pulse when the transmitted power is low are not due to self-excited loop (SEL) instabilities. The effect of the pressure detuning coincides with the peak power dissipation (red curve). The slow drop in frequency after $20 \mathrm{~ms}$ is due to a low frequency mechanical vibration (10-20 Hz) and a higher frequency mechanical oscillation $(\sim 200 \mathrm{~Hz})$ is also visible. The DQW PoP crab cavity's resonant frequency has a measured pressure sensitivity of $448 \mathrm{~Hz} / \mathrm{mbar}$ [4]. The observed pressure detuning during a quench of the DQW PoP cavity is $100-200 \mathrm{~Hz}$. This would give a pressure spike of 0.22 0.44 mbar, which is consistent with the value of 0.23 mbar predicted by the model in Sec. II B.

Figure 4 shows the dissipated power, $P_{C}$, in the cavity vs time. The dissipated power peaks at approximately $1.85 \mathrm{~kW}$ at a time of $31.16 \mathrm{~ms}$, which is consistent with the suspected pressure detuning spike. Unfortunately, there is no way to conclusively prove that the frequency spike in Fig. 4 is due to pressure detuning. However the peak in power dissipation occurring at the same time as the frequency spike strongly suggests that this is likely due to pressure detuning.

\section{Extrapolation to HL-LHC}

As previously stated, the DQW PoP crab cavity has a measured Lorentz force detuning factor of $-206 \mathrm{~Hz} / \mathrm{MV}^{2}$ and a pressure sensitivity of $448 \mathrm{~Hz} / \mathrm{mbar}$. By comparison, the dressed crab cavity that will be installed in SPS has a measured Lorentz force detuning factor of $-40 \mathrm{~Hz} / \mathrm{MV}^{2}$ and a pressure detuning sensitivity factor of $-0.103 \mathrm{~Hz} / \mathrm{mbar}$ [26]. These values are also expected to be the same for HL-LHC.

Based on these measured values and the measured frequency shift for the PoP cavity, we estimate a frequency shift for SPS/HL-LHC due to Lorentz force detuning of $\sim 460 \mathrm{~Hz}$ and a pressure detuning of $\sim 0.3-0.6 \mathrm{~Hz}$. For the PoP crab cavity, it was observed that Lorentz force detuning and its associated microphonics were the dominant mechanism of frequency detuning; for SPS/HL-LHC, it is expected to be even more dominant.

\section{E. Choice of quench parameters for simulations}

In reality, the dynamics governing the behavior of a quench is a very complex, multiphysics problem. As such, the quench parameters can vary drastically from one quench to another in the same structure. For example, it is known that the quench transition time can vary by several orders of magnitude, depending on the initial location of the quench, due to the local physical and thermal properties of the cavity as well as the cause of the quench. For HL-LHC, quenches which occur on the order of the bunch revolution period are considered to have the highest potential to result in significant beam loss because they will cause every bunch to be scattered onto different orbits; 
resulting in large betatron oscillations. For very fast quenches, only a few bunches are likely to be scattered, while all other bunches will remain on the reference orbit; thus only limited beam losses would be expected. Conversely, very slow quenches are also not a problem because feedback systems within the HL-LHC ring will correct the orbits of scattered bunches and any particle losses will occur over many turns, by which point the interlock system will have dumped the beam.

For the simulation studies described in the following sections, we choose the worst-case scenario for quench parameters and therefore choose a quench transition time of $\tau_{q}=10 \mu \mathrm{s}$, which results in the transverse deflecting voltage taking approximately 1 revolution period of the HL-LHC to go from nominal value to the post-quench value $(\sim 1 \mathrm{kV})$. We will use a Lorentz detuning factor which is 5 times larger than the expected value to allow a margin of safety in the simulations. Similarly we will also use a pressure detuning factor which is a factor of 5 larger as the detuning factor assumes a uniform pressure on the cavity surface, the effect of a nonuniform pressure is less predictable. For simulations at $4 \mathrm{~K}$, we use a significantly higher pressure detuning factor to provide a frequency shift of $4000 \mathrm{~Hz}$, which is consistent with the observed phase shift during a quench of the KEKB crab cavity [3].

\section{BEAM DYNAMICS STUDIES}

Particle tracking simulations were undertaken in two different ways. First, a method of transporting particles using sector maps produced by MAD-X [27] and a second method where the Matlab cavity simulation results are read into the tracking code SIXTRACK [28], in order to model the interaction between the cavity and the bunch train.

In these simulations, we assume that only one crab cavity has failed. In the cases where there are multiple crab cavities (such as for HL-LHC) we assume all the other cavities are ideal and do not have phase or amplitude jitter.

\section{A. Tracking with sector maps}

A map refers to a transformation which describes how the particle phase space distribution changes from one location in a beam line to a location further downstream. In circular machines, a one-turn map refers to the transformation of the phase space distribution when the bunch travels an entire revolution of the ring and a sector map refers to the transformation of the phase space over some part of the ring.

A first order map, often known as a transfer matrix, can be expressed in index notation as

$$
x_{i}^{(1)}=\sum_{j} R_{i j} x_{j}^{(0)},
$$

where the superscripts 0 and 1 refer to the initial and final coordinates respectively. For these studies, we consider the first and second order tensors, in which case, the transformation is written as

$$
x_{i}^{(1)}=\sum_{j} R_{i j} x_{j}^{(0)}+\sum_{j} \sum_{k} T_{i j k} x_{j}^{(0)} x_{k}^{(0)} .
$$

If we now use a second sector map to transform the beam from position " 1 " to position "2" and express in terms of $x^{(0)}$, we obtain

$$
\begin{aligned}
x_{i}^{(2)}= & \sum_{j} \sum_{k} R_{i j}^{(2)} R_{j k}^{(1)} x_{k}^{(0)} \\
& +\sum_{j} \sum_{k} T_{i j k}^{(2)}\left(\sum_{l} R_{j l}^{(1)} x_{l}^{(0)}\right)\left(\sum_{l} R_{k l}^{(1)} x_{l}^{(0)}\right) \\
& +\sum_{j} R_{i j}^{(2)}\left(\sum_{k} \sum_{l} T_{j k l}^{(1)} x_{k}^{(0)} x_{l}^{(0)}\right)+\mathcal{O}\left(x^{4}\right) .
\end{aligned}
$$

Note in Eq. (51) that tracking through multiple second (or higher) order maps introduces additional higher order terms. In the sector map tracking, we truncate to second order as these higher order terms are orders of magnitude smaller than the first and second order terms and storing these higher order terms will significantly increase the required computing time to track particles over many turns.

The particles are tracked from one crab cavity to the next (or to the interaction points IP1 and IP5) and each cavity is modeled as a thin kick by applying the angular deflection from the cavity to the beam at the longitudinal midpoint of the cavity. The tracking simulations using sector maps shown in this article track 3 particles, which initially lie on the reference orbit, but are longitudinally at the head, center, and tail of the bunch.

The use of sector maps is advantageous because it allows the particles to be tracked very quickly by avoiding the need to model every element in the beam line. However, in a ring this technique does not allow us to accurately predict beam losses as the particles are simply mapped from one location to another in a discrete manner. Sector map tracking was used to qualitatively study the cavity behavior and the interaction between the beam and the cavity.

\section{B. Particle tracking in SixTrack}

Particle tracking in SIXTRACK allows for a more detailed analysis of the beam dynamics and losses, but requires significantly more simulation time than the sector map method. It works by tracking the particles element-by-element through the lattice of the machines. Two versions of this code are available: The "standard" version that only considers the deterministic particle dynamics [28-30], and the "collimation" version [31] which also incorporates Monte-Carlo routines for scattering the particles in the collimator jaws. This scattering is important for understanding the spatial distribution of the losses, especially in the superconducting magnets. 
In general, the "standard" SIXTRACK version was used as no collimation setup was available for the SPS; here the losses were computed by comparing $\{x, y\}$ position of each particle to the aperture at the aperture bottlenecks. For the HL-LHC, where collimation input files are available [32-34], the collimation version of SIXTRACK was also run. The latter code also takes the material of the bottlenecks (i.e., the primary collimators) into account, allows for scattering or absorption of the particles using a MonteCarlo method, and computes a loss map along the ring. A comparison between the losses code that only takes the bottlenecks into account, and the SIXTRACK collimation version was performed. As shown in Fig. 15, the results are very similar, indicating that only considering the aperture bottlenecks is a reasonable approximation.

In the SIXTRACK simulations, each bunch in the bunch train is treated separately, and the cavity voltage and phase as a function of time are loaded from pre-calculated files using the dynamic kicks functionality (DYNK) [35,36], which updates the cavity parameters at the beginning of every turn. The precalculated files were prepared as described earlier in this paper.

All the simulations were run for a total of 120 turns, where the cavity parameters are kept constant at their "ideal" values for the first 20 (SPS) or 100 (HL-LHC) turns, before the mentioned files are loaded. Note that the plots only show what happened after the cavity parameters were "unlocked" and allowed to vary.

In the SixTrack studies, we neglect beam loading. For HLLHC, the bunch charge is approximately $18.4 \mathrm{nC}$ and the $\mathrm{rf}$ amplifier for the cavity can deliver a maximum of $80 \mathrm{~kW}$, from Eq. (40), we can estimate that for beam loading to be comparable to the maximum rf power, the full bunch train would need a transverse offset of approximately $4 \mathrm{~mm}$. In reality, the expected offset is estimated to be of the order of $100 \mu \mathrm{m}$. Furthermore, the assumption that the bunch train has a constant transverse offset is unphysical. In reality, the transverse motion of the beam undergoes betatron oscillations, which will cause the beam loading effect to cancel itself over approximately 3 revolutions of the ring.

For both the HL-LHC and SPS cases, the particles in each bunch were assumed to be distributed according to a correlated bivariate Gaussian with tails in each plane of the transverse phase space; the tails, representing $5 \%$ of the total bunch population, were taken to have the same distribution and were 1.8 times wider in $\left\{x, x^{\prime}\right\}$ and $\left\{y, y^{\prime}\right\}$ as described in [8,37]. The initial particle distribution was matched at the injection point to the transverse phase space using the given Twiss parameters, dispersion, orbit, and emittance at the injection point; for the transverse tails the phase space was scaled by the given factor.

For the longitudinal phase space, an uncorrelated bivariate Gaussian distribution was used; here the bunch length and energy spread were set to fill the bucket. The energy spread was adjusted by hand in order to minimize the oscillation in bunch length, something that occurs when the distribution is not perfectly matched. Some amount of this oscillation remained, however it is not likely to have any considerable impact on the results as the oscillation period (approximately $\frac{1}{2}$ the period of the synchrotron oscillations) is much slower than the processes under study. In order to avoid simulating particles outside of the rf bucket, when generating the longitudinal distribution we check that the initial energy and $z$-position of each generated particle was inside the bucket, and generated new longitudinal coordinates for any particles that did not pass this test until they did pass, effectively cropping the distribution at the bucket edge with an accept/reject Monte-Carlo generator.

In total, each bunch was represented by 60,000 particles, 40,000 for the core and 20,000 for the tail; the finer sampling of the tails were used in order to improve the beam loss estimates. Each bunch was generated from a separate random seed, and the bunches were different between the different machines but identical between different simulations of the same machine; i.e., the initial distribution of bunch 1 for HL-LHC was always the same, and so on for the rest of the bunches and for the SPS simulations. This allows comparison between the simulations, subtracting the statistical noise from the effect of the crab cavity.

\section{RESULTS}

\section{A. Benchmarking with KEKB results}

The motivation for this study was due to experimental measurements of the crab cavities installed at KEKB [3]. During a quench, KEKB observed the crab cavity phase shifting by up to $50^{\circ}$ within $50 \mu$ s. Based on the pressure detuning model described in Sec. II B, in order to achieve a phase shift of 50 degrees in $50 \mu \mathrm{s}$, a frequency shift of $3-4 \mathrm{kHz}$ is required (depending on parameters of the LLRF and vacuum systems). Assuming that the pressure sensitivity of the KEKB crab cavity is similar to the HL-LHC cavity, a pressure spike of the order of $10^{2}$ mbar would be required, which is consistent with the expected pressure spike from the model.

The quench presented in Ref. [3] shows that the klystron power begins to slowly increase approximately $800 \mu \mathrm{s}$ before the quench is detected and the rf is switched off. The beam is dumped approximately $400 \mu s$ after the $\mathrm{rf}$ is switched off. For the presented quench, appears that the pressure detuning rapidly increased at approximately the same time as the rf was switched off.

Due to the unpredictable nature of a quench, there are many variables, such as the location of the center of the quench, which can greatly alter the transition time of the quench and the time at which pressure detuning becomes significant.

In addition to the observed behavior due to a quench, microphonics were also observed at KEKB [3]. For the 


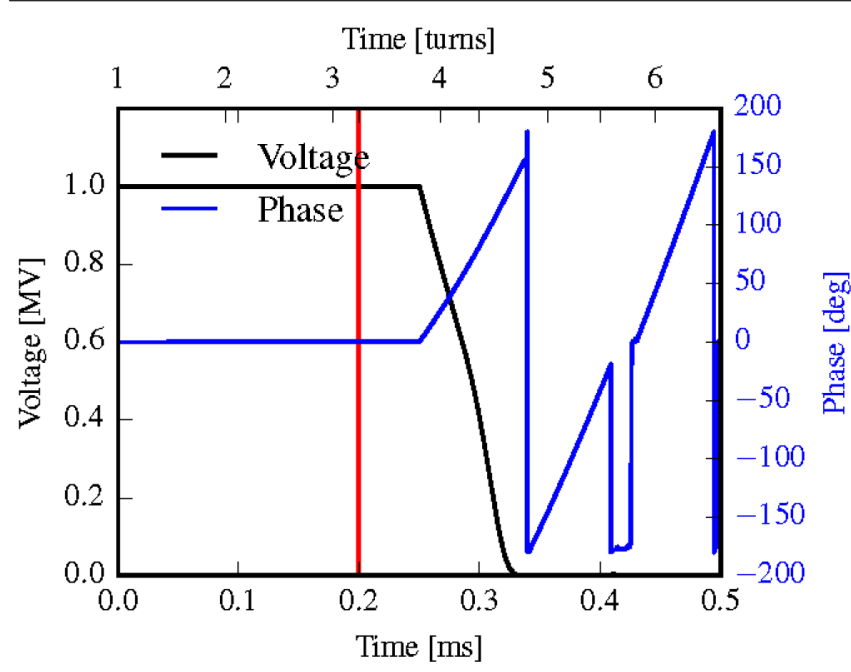

FIG. 5. Plot of the simulated cavity voltage and phase vs time during a quench for a KEKB-like cavity. The vertical red line represents the start time of the quench.

KEKB crab cavities the microphonics affects the cavity phase more than the amplitude and that it predominantly consists of a single frequency component. This is generally true for SRF cavities because as shown in Eq. (7), the rf phase is very sensitive to changes in frequency, but the amplitude is not.

Figure 5 shows the results of a simulation of a KEKBlike cavity during a quench. The simulation uses the HLLHC beam optics, but the KEKB crab cavity parameters from [3], summarized in Tables I and II. Hence the beam loading will not accurately represent the true KEKB system; but these studies still allow a qualitative interpretation of some of the key features observed in [3].

In the simulation, the frequency shift due to pressure detuning, which depends on the stiffness and geometry of the cavity, was assumed to be $\Delta f_{p}=4 \mathrm{kHz}$, but is consistent with measurements of other SRF structures, given that the boiling LHe causes a pressure increase on the

TABLE I. Key parameters of the SIXTRACK simulations for the three machines.

\begin{tabular}{lrccc}
\hline \hline & & SPS/55 & SPS/120 & HL-LHC \\
\hline Optics name & & Nom. Q26 & Nom. Q26 & v1.2 \\
Num. bunches & & 288 & 288 & 2810 \\
Energy $/ p^{+}$ & {$[\mathrm{GeV}]$} & 55 & 120 & 7000 \\
Rev. time & {$[\mu \mathrm{s}]$} & 9.7 & 9.7 & 88.9 \\
$\epsilon_{n} \mathrm{H} / \mathrm{V}$ & {$[\mu \mathrm{m}]$} & $3.1 / 2.8$ & $3.1 / 2.8$ & $2.5 / 2.5$ \\
Tune $Q_{x} / Q_{y}$ & & $26.13 /$ & $26.13 /$ & $62.31 /$ \\
& & 26.18 & 26.18 & 60.32 \\
Sync. tune & & 0.00805 & 0.00583 & 0.00212 \\
CC plane & & $\mathrm{V}$ & $\mathrm{V}$ & $\mathrm{V}$ \\
$\beta_{y}$ at CC & {$[\mathrm{m}]$} & 64.7 & 64.7 & 3760 \\
$\Delta y^{\prime}=\frac{q V}{E}$ & {$[\mu \mathrm{m}]$} & 55 & 25 & 0.43 \\
$\Delta y^{\prime} / \sqrt{\epsilon_{g} / \beta_{y}}$ & {$[\sigma]$} & 2.02 & 1.36 & 1.97 \\
\hline \hline
\end{tabular}

TABLE II. Comparison between the KEKB [3,38] and HL-LHC [1] crab cavities.

\begin{tabular}{lcc}
\hline \hline Cavity parameter & KEKB & HL-LHC \\
\hline Beam energy [GeV] & 8 & 7000 \\
Transverse voltage [MV] & 1 & 3 \\
Resonant frequency [MHz] & 509 & 400 \\
Transverse R/Q $[\Omega]$ & 50 & 400 \\
Superconducting $Q_{0}$ & $10^{9}$ & $10^{9}$ \\
Normal conducting $Q_{0}$ & $10^{3}$ & $10^{3}$ \\
$Q_{\mathrm{e}}$ & $1 \times 10^{5}$ & $5 \times 10^{5}$ \\
Operating temperature $[\mathrm{K}]$ & 4 & 2 \\
Quench transition time $[\mu \mathrm{s}]$ & 10 & 10 \\
LLRF parameters & & \\
Latency $[\mu$ s] & 1 & 1 \\
Digital refresh time $[\mathrm{ns}]$ & 25 & 25 \\
Proportional controller gain $\left(c_{p}\right)$ & 6.06 & 30.3 \\
Integral controller gain $\left(c_{i}\right)$ & $6.94 \times 10^{-7}$ & $3.47 \times 10^{-6}$ \\
Amplifier Q & 400 & 400 \\
Maximum tetrode power $[\mathrm{kW}]$ & 80 & 80 \\
Signal to noise ratio & 1000 & 1000 \\
Detuning parameters & & \\
Lorentz $\left(K_{L}\right)\left[\mathrm{Hz} / \mathrm{MV}{ }^{2}\right]$ & 200 & 200 \\
Pressure $\left(\Delta f_{p}\right)$ at $4 \mathrm{~K}[\mathrm{~Hz}]$ & 4000 & 4000 \\
Pressure $\left(\Delta f_{p}\right)$ at $2 \mathrm{~K}[\mathrm{~Hz}]$ & 100 & 100 \\
Microphonics $\left(\Delta f_{m}\right)[\mathrm{Hz}]$ & 2000 & 2000 \\
Mechanical frequency $\left(\omega_{m}\right)[\mathrm{Hz}]$ & 900 & 900 \\
\hline \hline
\end{tabular}

cavity of 0.1-1 Bar [13,14]. In Fig. 5, the discrete jumps in phase between $0.4-0.5 \mathrm{~ms}$ are due to beam loading. As the cavity voltage is very low, the changing voltage due to the beam can shift the phase rapidly.

The results from the simulations show that when the rf is switched off, which is also assumed to be approximately when the pressure detuning becomes significant, the phase shifts by $80^{\circ}$ in $50 \mu \mathrm{s}$; similar to the $50^{\circ}$ in $50 \mu$ s measured at KEKB. In addition to the consistency of the rate of change of phase between simulation and measurement, the ramp down of the voltage amplitude is also consistent. The time for the voltage to ramp down is approximately $50 \mu$ s for both cases and the "kink" on the curve is consistent; the kink is dependent on $Q_{L}$ and rate of change of $Q_{L}$ during a quench.

Figure 6 shows a simulation of the microphonics in a KEKB-like cavity. The voltage amplitude varies by approximately $1.5 \%$ and the time structure of the bunch train is observed due to beam loading. The frequency shift due to microphonics is assumed to be $\Delta f_{m}=2 \mathrm{kHz}$ and results in a phase oscillation of $\sim 4.5^{\circ}$; the KEKB studies measured a $4^{\circ}$ phase oscillation, showing good agreement.

\section{B. Parameter studies}

The benchmarking of the cavity quench model to measurements from the KEKB crab cavities has shown that the model can successfully reproduce the key features observed during a quench. However, this does not provide 


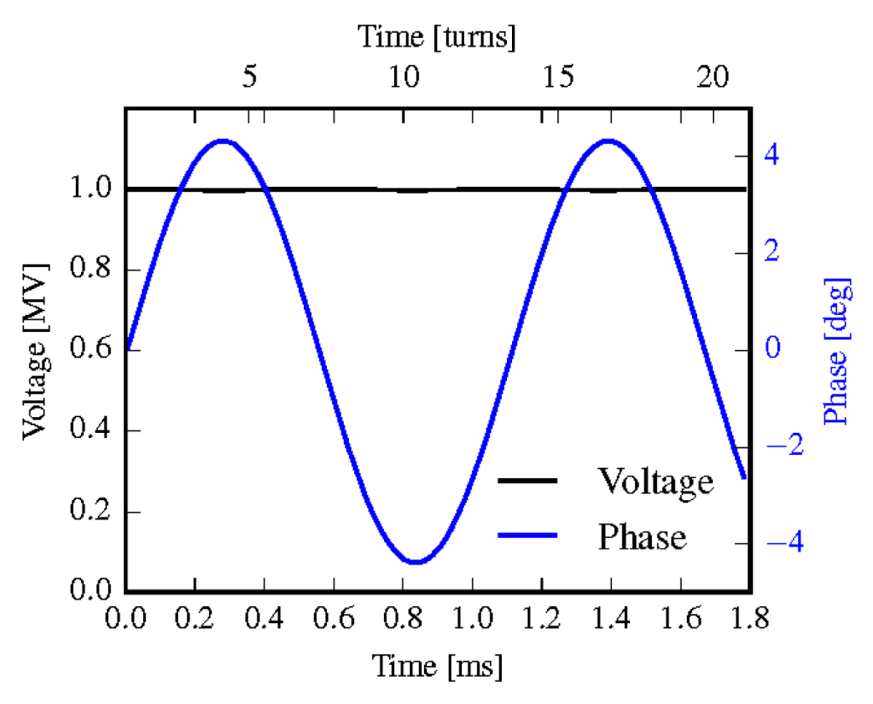

FIG. 6. Plot of the simulated cavity voltage and phase vs time under the influence of microphonics.

any insight into the factors which affect the cavity behavior during a quench. In order to determine which physical phenomena affect the cavity, a study was undertaken that compares the simulation results when different effects were included or omitted in the model. For the simulations in this study, microphonics will be neglected in order to make the quench behavior easier to see in plots. The cavity detuning is treated in exactly the same way for all simulations, although the magnitude of pressure detuning depends on the temperature of the LHe that we are simulating.

Table II shows the cavity, LLRF and quench parameters used during the studies in this paper. For these studies, the quenching cavity is taken to be the crab cavity further upstream of interaction point, IP1 (ATLAS), for Beam 2. The bunch positions are taken at the center of this cavity during tracking simulations.

\section{KEKB-like cavity}

Figure 7 shows the amplitude and phase of the KEKBlike cavity when the LHe temperature is $4 \mathrm{~K}$. This is a comparison of the effects of including beam loading (BL) in the simulation (BL on/off) and keeping the rf on or off during a quench (rf on/off). Beam loading causes rapid fluctuations in the cavity voltage phase after the quench if the rf is switched off. Other than this, beam loading has an extremely small effect on the cavity voltage because the rf system is able to compensate.

It can be seen that the change in phase during a quench is reduced if the $\mathrm{rf}$ is kept on during a quench (green line) compared to turning the rf off (black and red lines). Figure 8 shows the same comparison as Fig. 7, but the LHe temperature is $2 \mathrm{~K}$ and therefore superfluid.

By comparing the phase plots in Figs. 7 and 8, it is clear that the rapid phase shifts observed on the KEKB crab cavities is due to the LHe temperature and switching off the
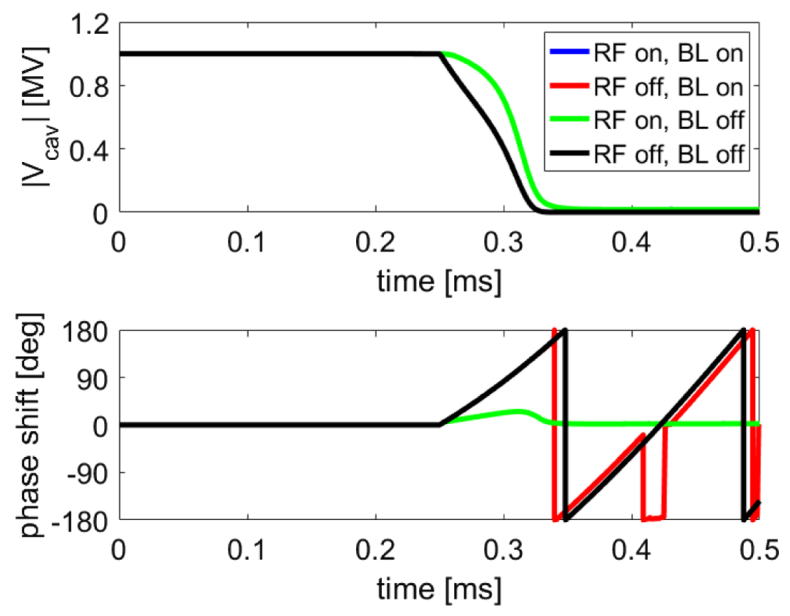

FIG. 7. Plots of cavity voltage amplitude (top) and phase (bottom) for a KEKB-like cavity at $4 \mathrm{~K}$ during a quench, comparing the effects of the rf system and beam loading.

rf during the quench. Figure 9 shows the transverse position of the centre of the bunches vs time with the rf kept on (blue) or switched off (red) when the quench is detected. The top plot shows the comparison when the LHe temperature is $4 \mathrm{~K}$ and the bottom plot when the LHe is at $2 \mathrm{~K}$. These plots use the sector map tracking method to provide a qualitative understanding.

In the top plot of Fig. 9, particles are cut if the transverse position exceeds $\pm 20 \mathrm{~mm}$; although this is not the actual aperture of the KEKB crab cavities, this is a reasonable aperture to assume for the optics used.

Table III shows the estimated number of particles which survive 10 turns after the quench for the KEKB case under different conditions. Only 3 particles are tracked per bunch for the sector map tracking, situated longitudinally at the head, middle, and tail of the bunch. Thus the values in
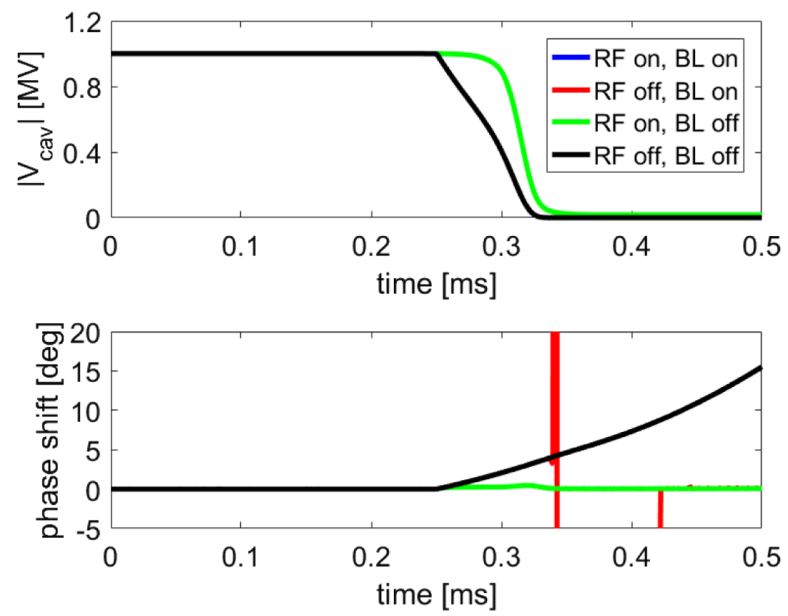

FIG. 8. Plots of cavity voltage amplitude (top) and phase (bottom) for a KEKB-like cavity at $2 \mathrm{~K}$ during a quench, comparing the effects of the rf system and beam loading. 

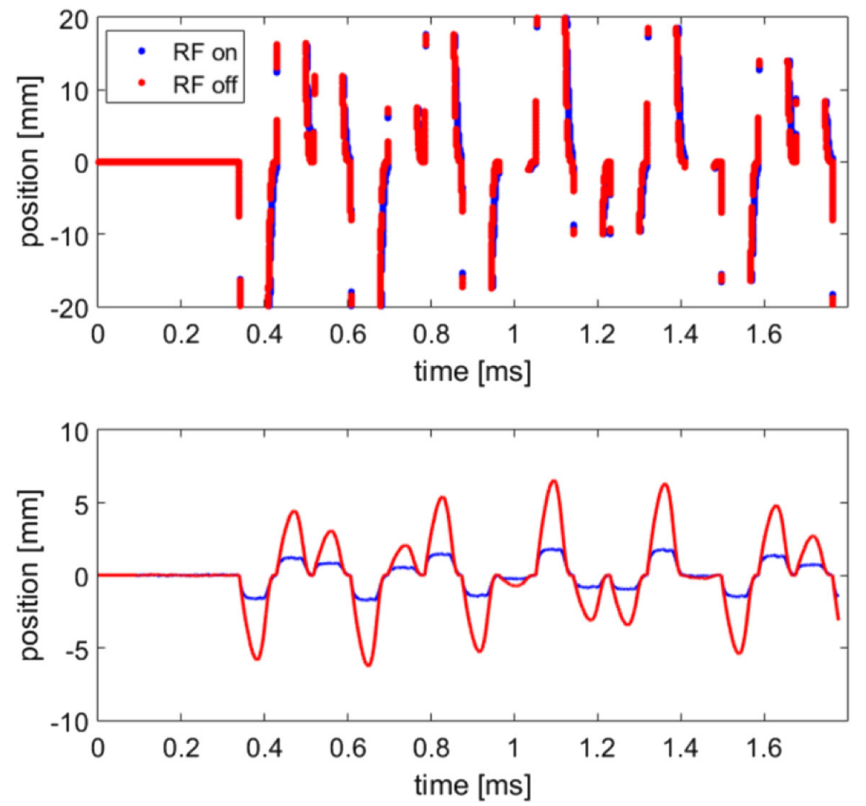

FIG. 9. Transverse beam position during a quench in $4 \mathrm{~K} \mathrm{LHe}$ (top) and $2 \mathrm{~K}$ LHe (bottom) for a KEKB-like cavity, with the rf kept on (blue) or switched off (red) when a quench is detected.

Table III are only meant to qualitatively understand the impact on the beam due to a crab cavity failure.

As expected, beam loading causes a negligible effect on the fraction of the beam which survives 10 turns. However, counterintuitively, keeping the rf on during a quench causes more particle losses than switching it off during a quench at both $2 \mathrm{~K}$ and $4 \mathrm{~K}$. This can be explained by inspection of Figs. 7 and 8. Although the rf reduces the phase shift during a quench, it also keeps the amplitude of the voltage higher for longer; hence providing more erroneous deflection to the bunch train for longer. Therefore for the KEKB case, it is preferable to protect the rf system and switch it off when a quench is detected.

\section{HL-LHC cavity}

The same study was repeated for the HL-LHC cavity, using the parameters in Table II. Figures 10 and 11 show the cavity voltage and phase when cooled in $4 \mathrm{~K}$ and $2 \mathrm{~K}$ LHe respectively, comparing the effects of beam loading and keeping the rf on or off during a quench.

We note once again that beam loading has very little effect on the cavity voltage except for causing jumps in the

TABLE III. Summary of fraction of particles surviving 10 turns after a quench of the KEKB-like cavity under different conditions.

\begin{tabular}{lcc}
\hline \hline Simulation & LHe at 2K & LHe at 4K \\
\hline rf on, BL on & $40.08 \%$ & $8.42 \%$ \\
rf off, BL on & $51.92 \%$ & $9.76 \%$ \\
rf on, BL off & $40.05 \%$ & $8.43 \%$ \\
rf off, BL off & $51.89 \%$ & $9.77 \%$ \\
\hline \hline
\end{tabular}
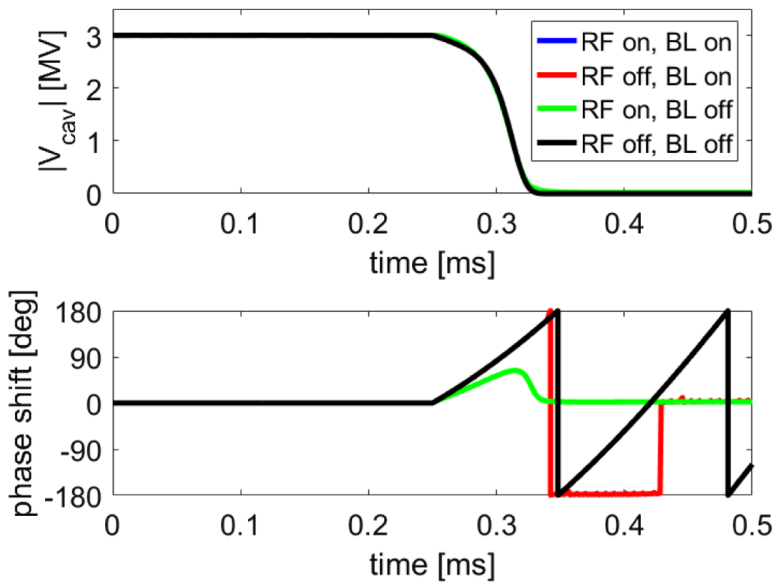

FIG. 10. Plots of cavity voltage amplitude (top) and phase (bottom) for an HL-LHC cavity at $4 \mathrm{~K}$ during a quench, comparing the effects of the rf system and beam loading.
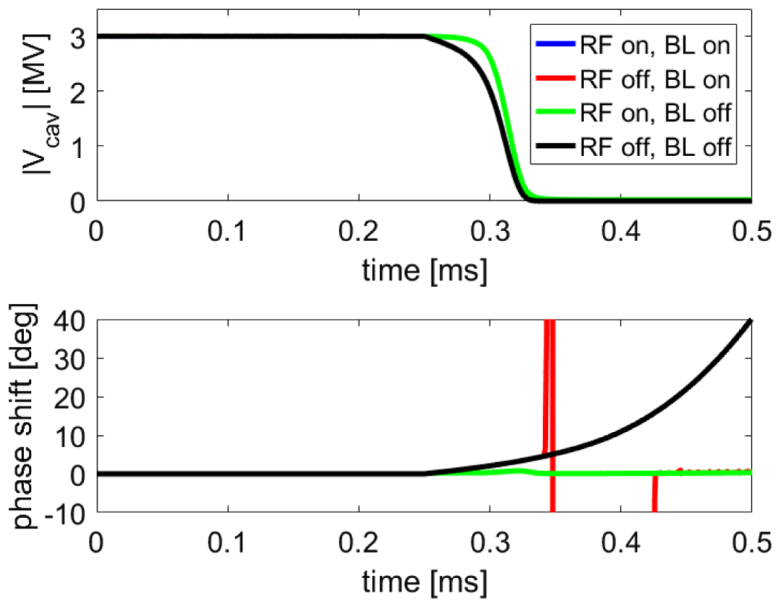

FIG. 11. Plots of cavity voltage amplitude (top) and phase (bottom) for an HL-LHC cavity at $2 \mathrm{~K}$ during a quench, comparing the effects of the rf system and beam loading.

cavity phase when the rf is switched off and only after the quench. Therefore for all further simulations, beam loading will be neglected. Figure 12 shows the cavity phase for the HL-LHC crab cavity during a quench under normal operating conditions. In the absence of microphonics, the phase changes by $<1^{\circ}$ with the rf kept on and the LHe temperature at $2 \mathrm{~K}$.

Figure 13 shows the transverse beam position during a quench when the LHe temperature is $4 \mathrm{~K}$ (top) and $2 \mathrm{~K}$ (bottom). Due to the much higher energy of the HL-LHC beam compared to KEKB, the beam, hence reference particle, experiences significantly less deflection.

\section{SixTrack simulation results}

For both the SPS and HL-LHC, 3 different cases were studied with SIXTRACK. They will hereafter be named as follows: 


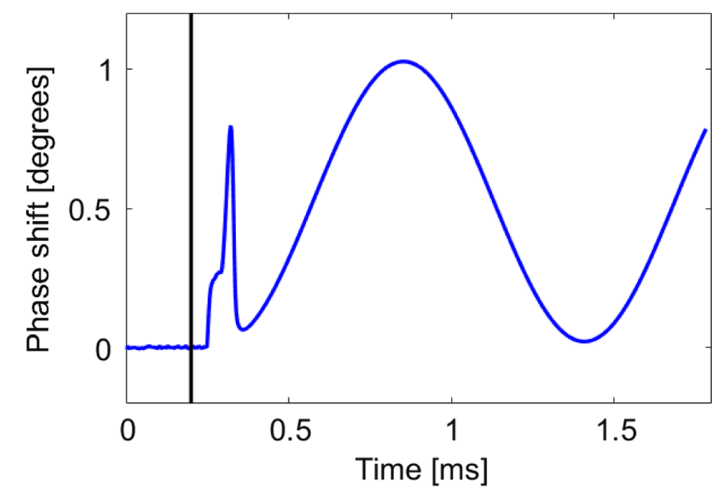

FIG. 12. Cavity phase vs time during a quench for an HL-LHC crab cavity under normal operating conditions.
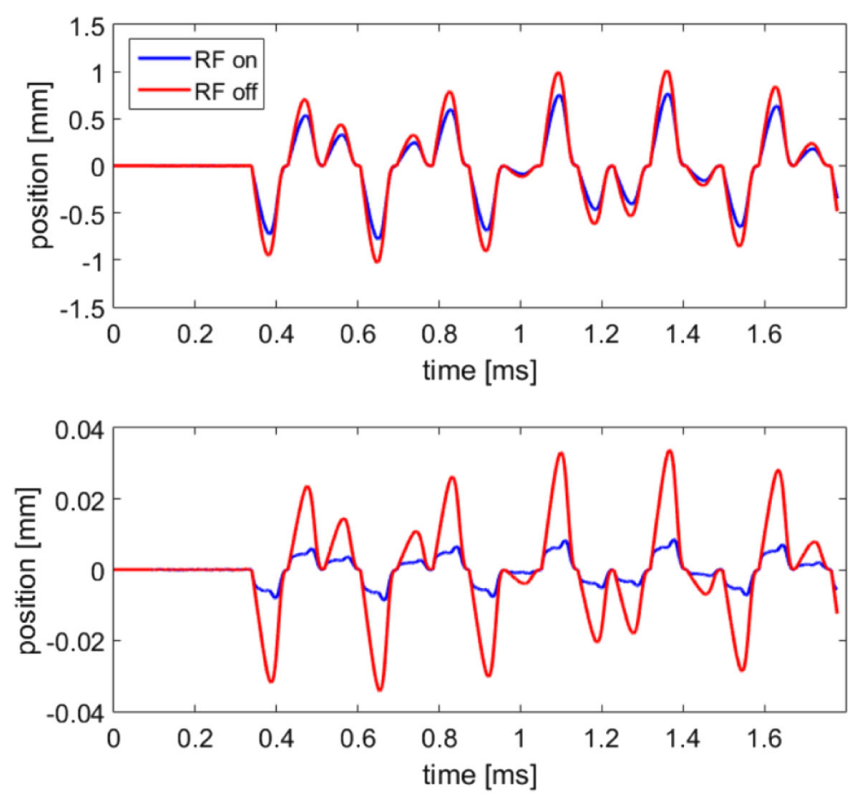

FIG. 13. Transverse beam position during a quench in $4 \mathrm{~K} \mathrm{LHe}$ (top) and $2 \mathrm{~K}$ LHe (bottom) for an HL-LHC cavity, with the rf kept on (blue) or switched off (red) when a quench is detected.

Const.-The crab cavity voltage amplitude and rf phase at the time of arrival for the ideal particle are constant throughout the simulation. This is meant as a reference of comparison for the other losses

NoFail.-The crab cavity voltage and phase are constant for 20 (SPS) or 100 (HL-LHC) turns in the beginning of the simulation in order to make sure that the beam has stabilized, and then follow a set of precalculated values corresponding to a cavity experiencing microphonics but no quench, being controlled by an LLRF controller for the remaining 100 or 20 turns. The modeled cavity is a LHC-type cavity at $2 \mathrm{~K}$, as described in Table II.

Fail.- The crab cavity voltage and phase are constant in the beginning of the simulation, and later follow a set of precalculated values corresponding to a cavity experiencing microphonics and then quenching with pressure detuning. The quench starts at $200 \mu \mathrm{s}$ after the "unlocking" of the crab cavity, and the pressure detuning occurs after $250 \mu \mathrm{s}$. The modeled cavity is a LHC-type cavity at $2 \mathrm{~K}$, as described in Table II. In all cases, the initial voltage and phase of the controlled CC was set to $3 \mathrm{MV}$ and $0^{\circ}$ respectively. The time-development of the rf voltage and phase are shown in Figs. 14 (LHC) and 17 (SPS), for both the NoFail and Fail scenarios. These plots start from the time where the controlled cavity is "unlocked," which is considered as $t=0$ and the beginning of turn 1. As shown in the plots, in the NoFail scenario the voltage remains constant at $3 \mathrm{MV} \pm 3 \mathrm{kV}$, while the swings within $\pm 2^{\circ}$ due to microphonics, both for the SPS and LHC cases; for the Fail scenario we see the same behavior up to $200 \mu$ s when the quench happens, the voltage then stays approximately stable for $80 \mu$ s until it drops to zero within $60 \mu \mathrm{s}$, while the phase has an excursion up to $6.7^{\circ}$ due to a combination of the pressure and Lorentz detuning, and microphonics.

\section{HL-LHC machine}

For the LHC, the cavity parameters were always constant for the first 100 turns, and then controlled by the LLRF system model for the last 20 turns. The voltage and phase during these last 20 turns of the Fail- and NoFail cases are shown in Fig. 14.

HL-LHC version 1.2 optics and sequence for beam 1 was used [1,39], which includes 4 cavities per beam per IP per side. Only one of the crab cavities, named "ACFCA.AL1.B1", which is the cavity on beam 1 "upstream" of IP1 that is closest to the IP, was controlled by the LLRF system and experienced the quench. All other cavities were assumed to have a constant voltage and phase; the other upstream cavities have a voltage of 3.0 MV, while for the downstream cavities the voltage is matched using MadX [27] to 2.871 MV for IP1 and 2.902 MV for IP5, in order to minimize orbit error through the ring during normal operation.

As mentioned previously, both collimation SIXTRACK and the standard SIXTRACK with an external aperture check was used to simulate the Fail and NoFail scenarios. This allowed comparing the losses between the two cases, checking that the specially developed external aperture check was functioning correctly, as the use of this code was a necessity for the SPS simulations. Using the external aperture check, the particle population was trimmed at the three primary collimators "TCP.D617.b1," "TCP.C617.b1," and "TCP.B617.b1," which are located in IP7 and have an aperture of $5.7 \sigma$ relative to a normalized emittance of $3.5 \mu \mathrm{m}$; equivalently, the collimator aperture is $6.74 \sigma$ relative to the actual normalized beam emittance of $2.5 \mu \mathrm{m}$. As seen in Fig. 15 (right), the two methods are quite comparable, with the external aperture check predicting slightly larger losses than the collimation version. This is expected as the external check does not take particle scattering into account, so that all particles touching the collimator are lost. Furthermore, if the material of the primary collimators in the collimation simulation is changed 

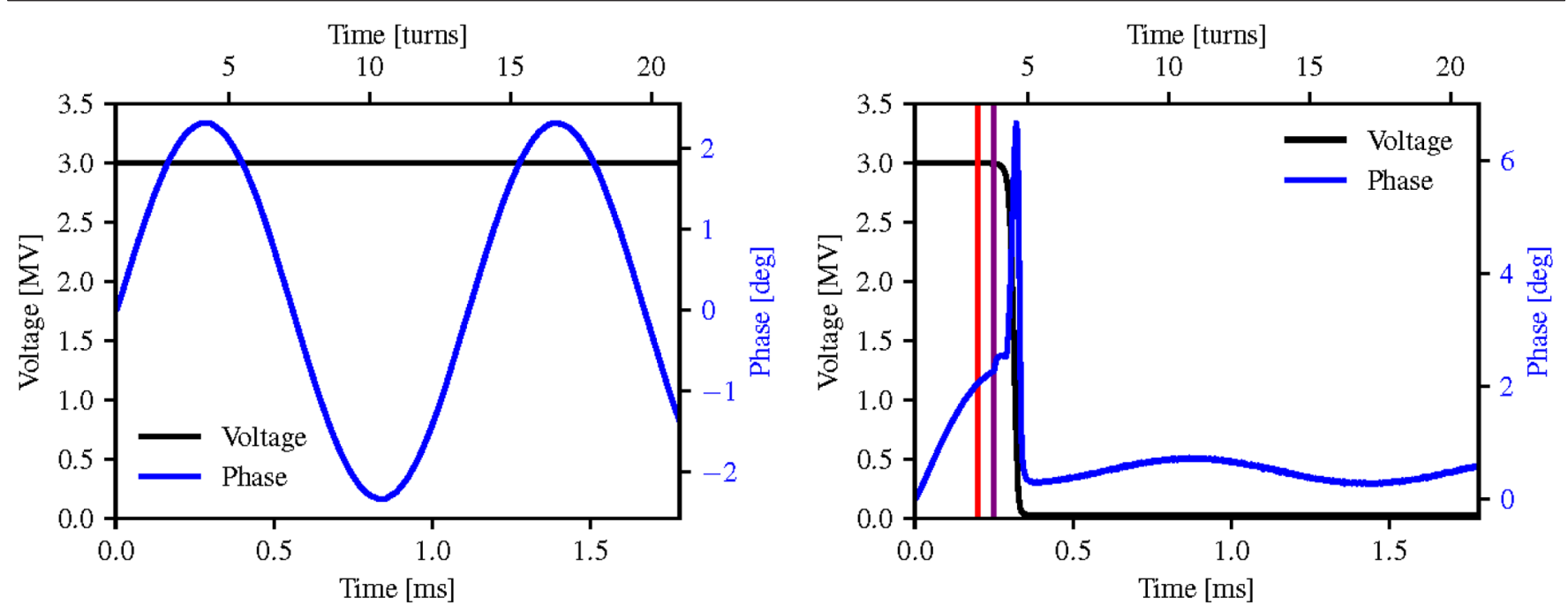

FIG. 14. Crab cavity voltage and phase as a function of time for the HL-LHC, both NoFail (left) and Fail(right) as defined in Sec. V C. For the Fail scenario, the first vertical line (red) marks the beginning of the quench, and the second line (purple) the time when pressure detuning becomes important.
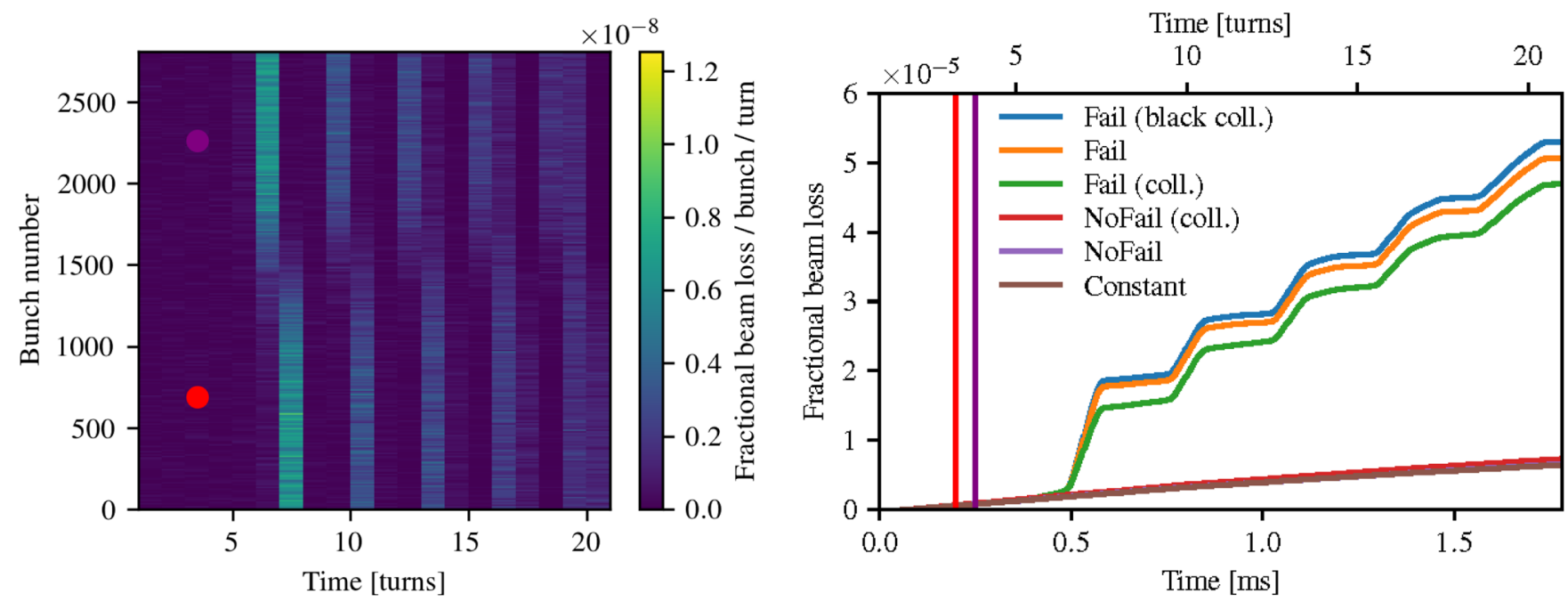

FIG. 15. Losses in the HL-LHC as a function of time; both instantaneous losses (left) for the Fail scenario, and cumulative losses comparing different scenarios and simulation techniques (right). The first vertical circle or line (red) marks the beginning of the quench, and the second circle or line (purple) the time when pressure detuning becomes important for the Fail scenario.

from carbon to a perfect black absorber, the predicted losses are larger than those predicted by the external aperture check. This is also expected since the collimation simulation takes the length of the collimators into account, while the external aperture check only checks the losses at a single plane. Finally, the losses are found to be on the order of $3 \times 10^{-5}$ of the full beam 10 turns after the failure, which is comparable to what has been seen in similar simulations previously $[33,40]$. This level of losses would be survivable for the collimation system [8,41]; however this is greatly influenced by the betatron tune and the phase advance between the failing crab cavity and the primary collimators [33].

As seen in the upper plots of Fig. 16, the cavity failure creates a movement of up to $\approx \pm 50 \mu \mathrm{m}$ in the vertical plane at the point of the cavity. As expected, the frequency of the beam position oscillation is the vertical betatron tune; and as seen in Fig. 15 (left), this frequency is also observed in the time profile of the losses, where the beam touches a collimator jaw every 3 turns. The centroid motion is caused by the phase error while the cavity voltage has not yet dropped to zero; however as this phase error is on the order of a few degrees, we observe a relatively small motion. In the horizontal plane, the centroid motion is only $\approx \pm 2 \mu \mathrm{m}$; this motion is caused by coupling from the vertical plane.

For the nonfailed case there is still a movement up to $\approx \pm 20 \mu \mathrm{m}$ due to the phase variation.

For the vertical beam size, shown in Fig. 16 (bottom), we see that it increases from approximately $1.2 \mathrm{~mm}$ to $1.45 \mathrm{~mm}$, 

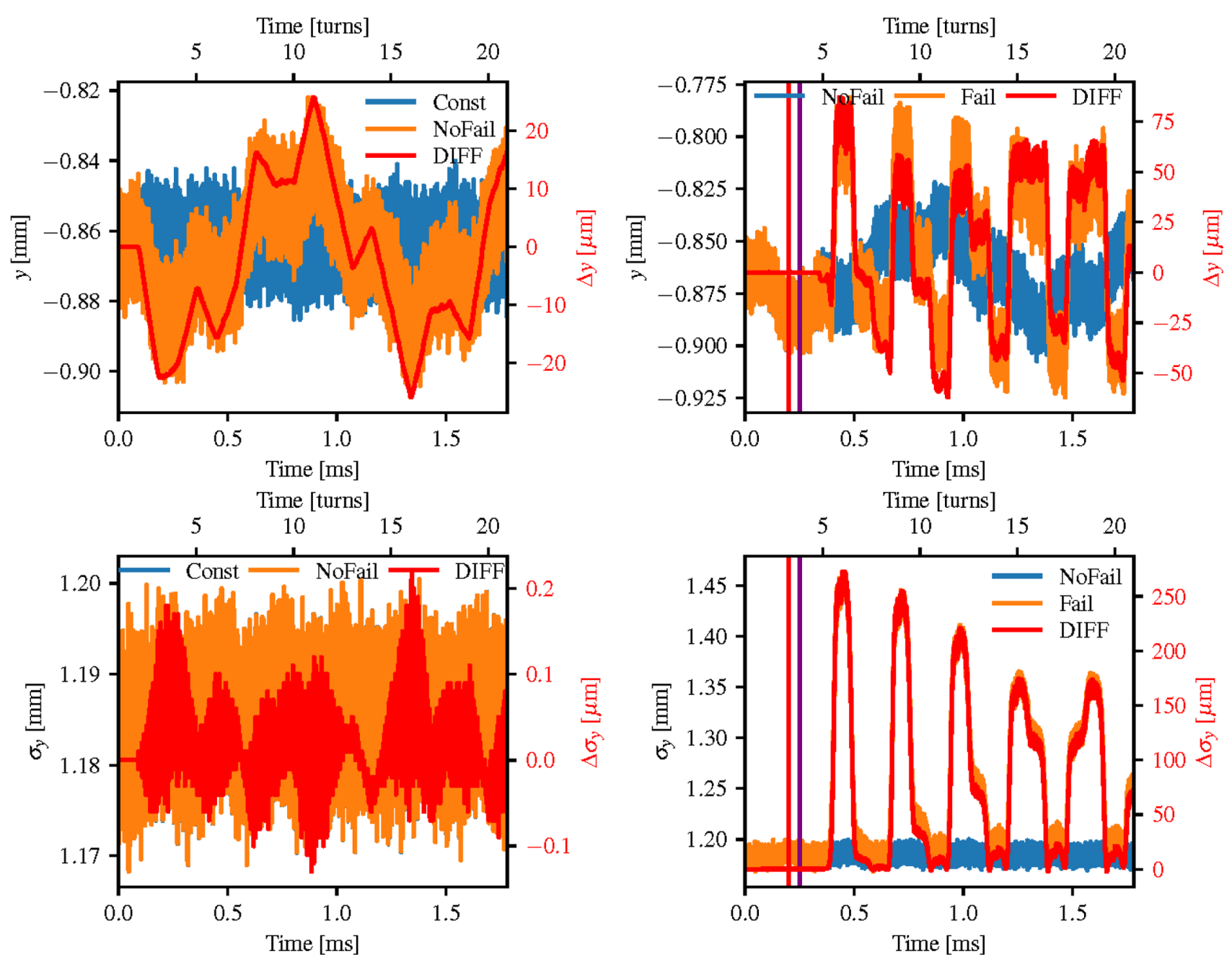

FIG. 16. Vertical beam position (top) and size (bottom) at the controlled crab cavity in the LHC, comparing scenarios NoFail vs Const (left) and Fail vs NoFail (right). For the Fail scenario, the first vertical line (red) marks the beginning of the quench, and the second line (purple) the time when pressure detuning becomes important.

an increase of $20 \%$. As discussed in more detail in the analysis of the SPS data, this is caused by different $z$-slices of the bunch seeing a different kick due to the noncancellation between the cavities creating and closing the bump, and are thus traveling on different trajectories. This results in the projection of the bunch onto the $y$-axis being wider, and thus a larger vertical beam size. The horizontal beam size remains constant within $1 \mu \mathrm{m}$ in all cases.

\section{SPS machine}

The crab cavities have never been used in proton machines and is therefore crucial to ensure that there will be no detrimental side effect for the beam in the HL-LHC machine. With this in mind, a set of prototype vertical crab cavities have been installed first in the SPS, and served as a test-bed between May to November 2018.

Prior to the crab cavity installation, dedicated machine development (MD) studies were undertaken in order to ensure good understanding of the SPS machine and the limitation of its instruments. Due to the limited available time for MDs, good preparation and planning of the studies was essential.

The quenching of the SPS crab cavity was studied using the nominal (Q26) SPS optics, the main parameters of which are summarized in Table I. The emittance was chosen based on the initial emittance at $55 \mathrm{GeV}$ [42]. Two different beam energies were studied, $55 \mathrm{GeV}$ and $120 \mathrm{GeV}$, and for each energy, 3 simulations were performed as discussed above. The controlled cavity was the first crab cavity along the ring, and the second one, installed $1.05 \mathrm{~m}$ later, was held a constant voltage of 3.0 MV and constant phase of $0^{\circ}$.

In order to study the losses in the SPS, the two aperture bottlenecks, the momentum scraper "TIDP" and a beam dump "TIDV," were included as described above. The $\{\mathrm{H}, \mathrm{V}\}$ apertures of these bottlenecks are $\{41,15\} \mathrm{mm}$ for the momentum scraper, which corresponds to a relative aperture of $\{19.8,13.06\} \sigma$; and $\{42.5,20.4\} \mathrm{mm}$ for the 

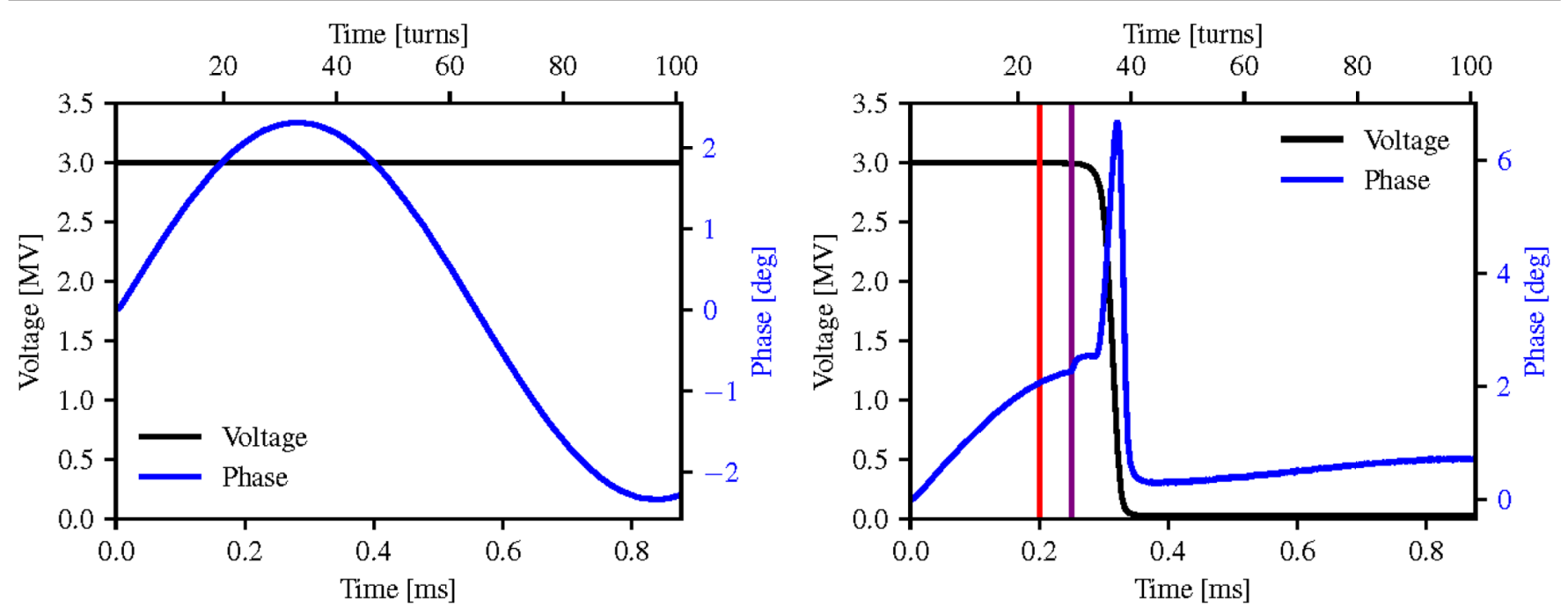

FIG. 17. Crab cavity voltage and phase as a function of time for the SPS, both NoFail (left) and Fail (right) as defined in Sec. V C. For the Fail scenario, the first vertical line (red) marks the beginning of the quench, and the second line (purple) the time when pressure detuning becomes important.

beam dump, which corresponds to $\{34.77,10.48\} \sigma$. The listed relative aperture values are calculated for the $55 \mathrm{GeV}$ case; for the $120 \mathrm{GeV}$ case the geometrical emittance is smaller and thus the relative aperture is even larger. No losses were observed from either the core or the halo, in either aperture bottlenecks.

From Fig. 18 (top left), we see that in the NoFail case (orange line) there is a small oscillation, which is due to the phase error caused by the microphonics which causes the orbit to change adiabatically; the period is approximately the same as the phase oscillation of cavity. Comparing the Fail and NoFail (top right plot), we can see that soon after the point where the cavity quenches, the amplitude of the vertical oscillation increases rapidly. Note that even for this case there are no losses observed in the SPS simulation, as the apertures bottlenecks are very large.

Unlike the vertical case, the horizontal centroid is constant in time in both Const and NoFail cases. In the Fail case, some very small oscillation is observed also in the horizontal centroid; this is due to the coupling between the vertical and horizontal planes that is introduced by the sextupoles installed in the SPS lattice.

There is a clear increase in the vertical beam size when the cavity quenches, as shown in Fig. 18 (bottom right); while for the NoFail there is little effect of the phase oscillation (bottom left plot). The oscillation in the Fail case is due to the head and tail of the bunch having a similar $y$ position. This is confirmed and explained by the evolution of the bunch shape shown in Fig. 19.

The frequency and amplitude modulation of the oscillations of the vertical beam size observed in Fig. 18 (bottom right) after the quench is due to the $z$-dependence of the closed orbit, which for a given particle moves with the synchrotron oscillation. Particles that before the quench were at the head or tail of the bunch, after the quench found themselves far away from their closed orbit, and were thus effectively injected into a large amplitude betatron oscillation around this new closed orbit. When the head and tail are opposite in phase of their betatron motions, this results in large $\sigma_{y}$ when they are furthest away from $y=0$ (Fig. 19, turn 40), and small $\sigma_{y}$ when they are both close to $y=0$ (Fig. 19, turn 42). This causes the fast oscillation with frequency equal to the betatron frequency, as seen in Figure 18 (bottom right). As the particles that were originally (before the quench) at the head and tail drift toward $z=0$ due to the synchrotron oscillation, their closed orbit moves toward $y=y^{\prime}=0$, adiabatically "dragging" the particles along. This means that their $y$-position is now only caused by the betatron oscillation, with little addition contribution from the orbit. At the same time, the particles that were originally close to $z=0$ are also moved adiabatically outward in $\left\{y, y^{\prime}\right\}$ phase space, resulting in the betatron amplitudes not increasing as much. This causes the modulation of the amplitude of the oscillation, which is four times the frequency of the synchrotron tune.

For the horizontal beam size no clear effect of the failure is observed, apart from a very small oscillation which is most likely due to the initial distribution not being absolutely perfectly matched.

Similar behavior is observed between the $55 \mathrm{GeV}$ and $120 \mathrm{GeV}$ cases. In both cases, the optics and thus the betatron tune is the same, which leads to the oscillations of the centroids staying in phase. The main difference is, as expected, the amplitude of the oscillations and beam sizes, as they scale linearly with energy. The other difference is the synchrotron tune, which is slightly faster for the $55 \mathrm{GeV}$ case. This also affects the oscillation of $\sigma_{y}$, where the "minimum" that is discussed above comes earlier for the $55 \mathrm{GeV}$ case; this is consistent with the explanation of this effect. 

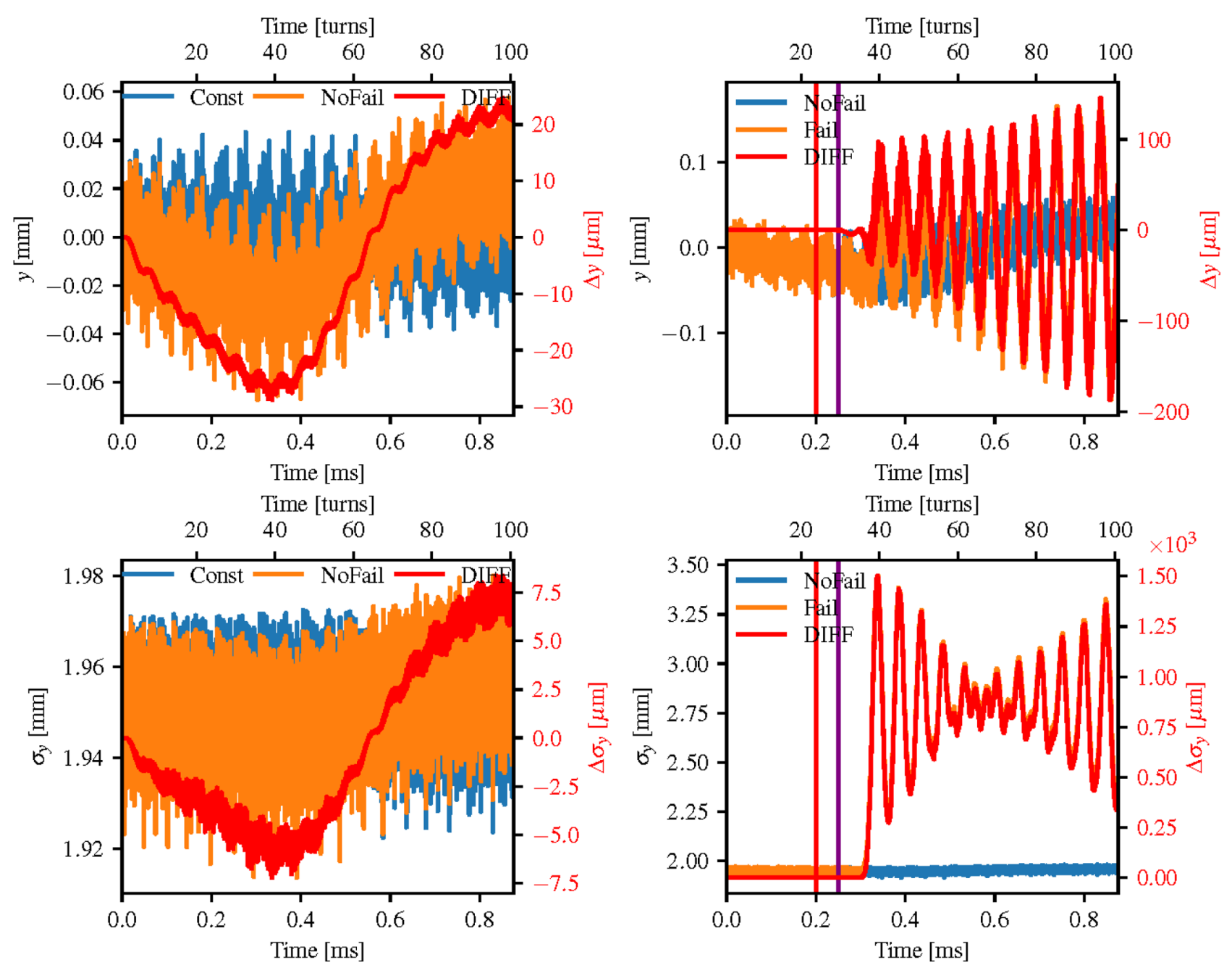

FIG. 18. Vertical beam position (top) and size (bottom) at the controlled crab cavity in the SPS/55 GeV, comparing NoFail vs Const (left) and Fail vs NoFail (right). For the Fail scenario, the first vertical line (red) marks the beginning of the quench, and the second line (purple) the time when pressure detuning becomes important.

\section{Comparison of the LHC and the SPS}

The observed centroid motion of the bunch is due to coherent betatron motion; and as expected, the frequency of the centroid motion is equal to the fractional tune. This was confirmed by a bunch-by-bunch FFT on the data. Given the difference in the fractional part of the betatron tunes (see Table I), this causes the centroid motion as a function of the turn number to be much faster in the LHC case.

The crab cavity failure in the SPS case is seen more gradually by the beam than for the LHC case, as the number of revolutions during the voltage collapse is larger (the SPS voltage drops to $0 \mathrm{~V}$ within 8 turns, as opposed to less than 1 turn in the LHC case, see Figs. 14 and 17).

Note that while the number of turns tracked after the crab cavity failure is much larger in the SPS (100) than for the LHC (20), the difference in revolution time (see Table I) causes the actual time from the start of the quench to the end of the simulation to be much larger in the LHC case (LHC: $1.5 \mathrm{~ms}$, SPS: $0.5 \mathrm{~ms}$ )
Comparing the effect of the maximum kick $(V=$ 3.0 MV) on the beam, from Table I we see that for the three cases the maximum kick $\Delta y^{\prime}$ is very different for the different energies and thus beam rigidity. However the betatron functions in the relevant plane are much higher for the LHC case than for SPS, resulting in a magnified effect on the beam. Thus when comparing the maximum kick $\Delta y^{\prime}$ to $1 \sigma$ of angular displacement $\sqrt{\epsilon_{g} / \beta_{\text {twiss }}}$, the resulting normalized kicks are very comparable between the 3 cases. However since in the SPS case the aperture is much larger, we only see losses in the HL-LHC case.

\section{CONCLUSIONS AND SUMMARY}

In this article we present a model of a rf cavity and include models of beam loading, the low-level rf system, detuning mechanisms, and superconducting quenches. These results were benchmarked against experimental measurements of the KEKB crab cavities. After successfully reproducing the 

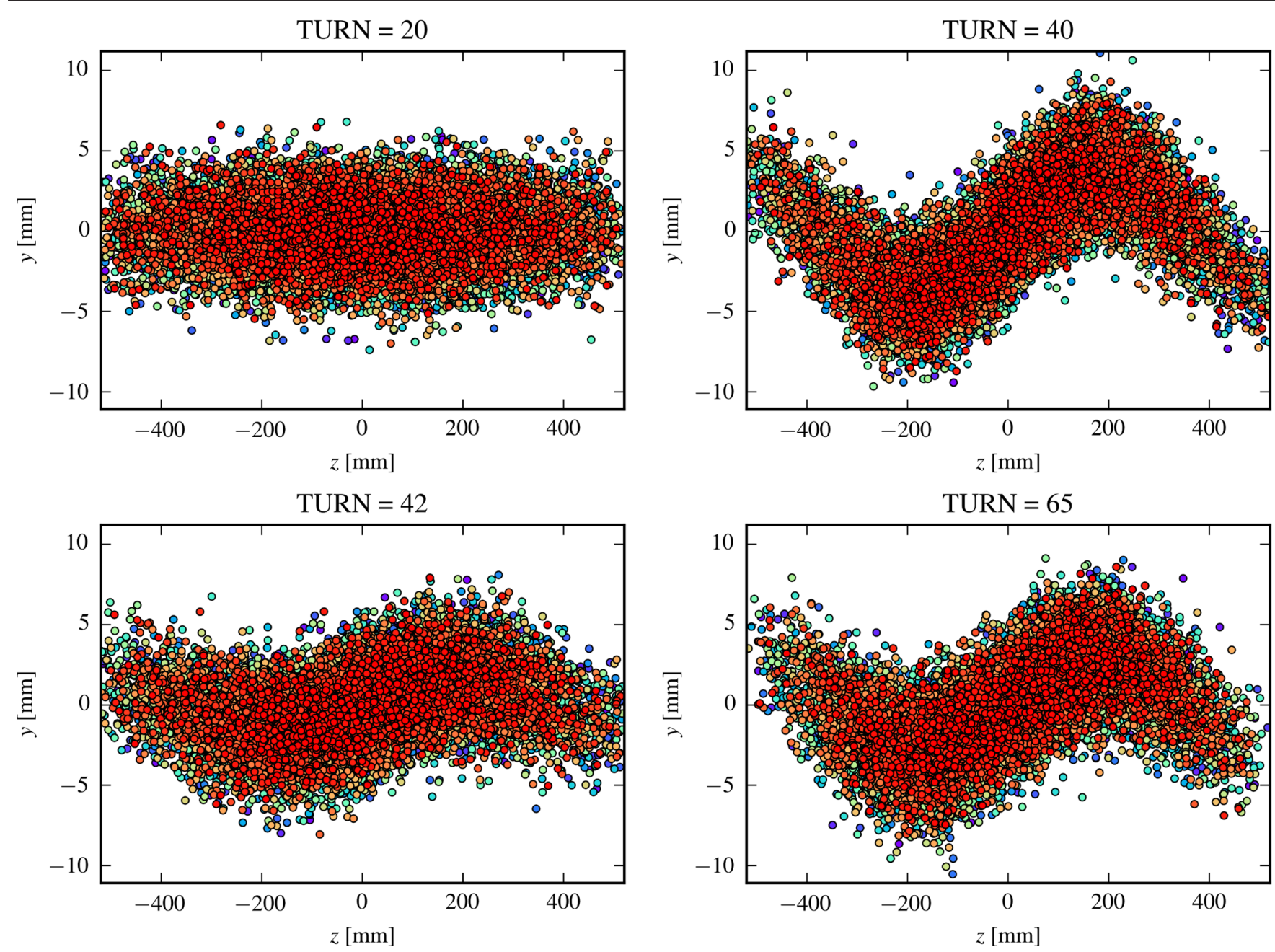

FIG. 19. Projection of the particle population of bunch 1 into the $\{z, y\}$ plane at 4 characteristic points in time, for the SPS at 55 GeV failure case at the point of the crab cavity under study. Upper left: Turn 20, before the quench; Upper right: Turn 40, near the top of the first peak seen in the left-hand plots of Fig. 18; Lower left: Turn 42, near the bottom of the first minimum just after that peak; Lower right: Turn 65 , near the stable region seen around $0.6 \mathrm{~ms}$, where the $y$ projection is quite stable between different turns.

key features from the KEKB crab cavity studies, parameter studies were undertaken to identify the causes of the rapid phase shifts observed at KEKB. The same parameter study was also undertaken for the HL-LHC crab cavity to model how it would behave during a quench under different conditions; this is essential for designing suitable machine protection for the crab cavity system.

Full tracking studies with SIXTRACK were undertaken to obtain a quantitative understanding of the expected beam losses in HL-LHC and SPS due to a quench of one of the crab cavities as well as the evolution of the beam parameters. The results show the beam loss is negligible for SPS and HL-LHC because the studies of quench dynamics and rf have indicated that the crab cavities would not be able to detune fast enough to deflect the beam before it is dumped.

Further studies are envisaged to use the cavity model described to study quenches of the HL-LHC accelerating cavities and has been integrated into SiXTrack as a module for other applications. This now allows SiXTRACK to communicate in a general way with external codes and files.

\section{ACKNOWLEDGMENTS}

The authors would like to thank Dr. Rama Calaga and Dr. Philippe Baudrenghien for their useful suggestions and insight. The studies presented have been funded by CERN as well as STFC Grants No. ST/N001575/1 and No. ST/ N001621/1.

[1] G. Apollinari, I. Béjar Alonso, O. Brüning, M. Lamont, and L. Rossi, High-Luminosity Large Hadron Collider (HL-LHC): Preliminary design report, CERN Yellow Reports: Monographs (CERN, Geneva, 2015), https:// cds.cern.ch/record/2116337. 
[2] J. Wenninger, Machine protection and operation for LHC, CERN Yellow Reports (CERN, Geneva, 2016), Vol. 2, p. 377.

[3] K. Nakanishi, Y. Funakoshi, and M. Tobiyama, Beam behavior due to crab cavity breakdowns, in Proceedings of the International Particle Accelerator Conference, Kyoto, Japan (ICR, Kyoto, 2010).

[4] B. Xiao, L. Alberty, S. Belomestnykh, I. Ben-Zvi, R. Calaga, C. Cullen, O. Capatina, L. Hammons, Z. Li, C. Marques, J. Skaritka, S. Verdu-Andres, and Q. Wu, Design, prototyping, and testing of a compact superconducting double quarter wave crab cavity, Phys. Rev. ST Accel. Beams 18, 041004 (2015).

[5] S. U. De Silva and J. R. Delayen, Cryogenic test of a proof-of-principle superconducting rf-dipole deflecting and crabbing cavity, Phys. Rev. ST Accel. Beams 16, 082001 (2013).

[6] S. U. De Silva and J. R. Delayen, Design evolution and properties of superconducting parallel-bar rf-dipole deflecting and crabbing cavities, Phys. Rev. ST Accel. Beams 16, 012004 (2013).

[7] B. Hall, G. Burt, R. Apsimon, C. J. Lingwood, A. Tutte, A. Grudiev, A. Macpherson, M. Navarro-Tapia, R. Calaga, K. G. Hernández-Chahín, R. B. Appleby, and P. Goudket, Design and testing of a four rod crab cavity for High Luminosity LHC, Phys. Rev. Accel. Beams 20, 012001 (2017).

[8] A. Santamaría García, K. Sjobak, R. Bruce, H. Burkhardt, F. Cerutti, R. Kwee-Hinzmann, and A. Lechner, Machine and experiment protection from fast crab cavity failures in the HL-LHC, IPAC'16 conference proceedings, Busan, Korea (2016), pp. 1485-1488, http://inspirehep.net/record/ 1469936/files/tupmw025.pdf.

[9] A. C. Dexter, G. Burt, and R. Apsimon, Active lower order mode damping for the four rod LHC crab cavity, Nucl. Instrum. Methods Phys. Res., Sect. A 844, 62 (2017).

[10] R. Apsimon and G. Burt, An improved equivalent circuit model of a four rod deflecting cavity, Nucl. Instrum. Methods Phys. Res., Sect. A 847, 52 (2017).

[11] J. Corno, C. de Falco, H. De Gersem, and S. Schöps, Isogeometric simulation of Lorentz detuning in superconducting accelerator cavities, Comput. Phys. Commun. 201, 1 (2016).

[12] J. E. Jensen, W. A. Tuttle, R. B. Stewart, and H. Brechna, Selected cryogenic data notebook, BNL Internal Report No. 10200-R, 1980.

[13] A. Neumann, W. Anders, O. Kugeler, and J. Knobloch, Analysis and active compensation of microphonics in continuous wave narrow-bandwidth superconducting cavities, Phys. Rev. ST Accel. Beams 13, 082001 (2010).

[14] M. H. Awida, I. Gonin, D. Passarelli, A. Sukanov, T. Khabiboulline, and V. Yakovlev, Multiphysics analysis of frequency detuning in superconducting RF cavities for proton particle accelerators, 2015 IEEE MTT-S International Conference on Numerical Electromagnetic and Multiphysics Modeling and Optimization (NEMO), pp. 1-3, http://lss .fnal.gov/archive/2015/conf/fermilab-conf-15-616-td.pdf.

[15] J. R. Delayen, Ponderomotive instabilities and microphonics —a tutorial, Physica C (Amsterdam) 441, 1 (2006).
[16] K. Sjobak, R. Bruce, H. Burkhardt, R. Kwee-Hinzmann, A. Macpherson, and A. Santamaría García, Time scale of crab cavity failures relevant for high luminosity LHC, IPAC'16 conference proceedings, Busan, Korea (2016), pp. 4196-4199, http://inspirehep.net/record/1470750/files/ thpoy043.pdf.

[17] P. Baudrenghien, LLRF for crab cavities, 2nd Joint HiLumi LHC-LARP Annual meeting, Frascati, Italy (2012), https://indico.cern.ch/event/183635/contributions/ 318488/attachments/250935/350850/CCLLRFv2.pdf.

[18] P. Baudrenghien et al., Functional specifications of the LHC prototype crab cavity system, CERN Report No. CERN-ACC-NOTE-2013-003, 2013.

[19] W. K. H. Panofsky and W. A. Wenzel, Some considerations concerning the transverse deflection of charged particles in radio-frequency fields, Rev. Sci. Instrum. 27, 967 (1956).

[20] D. R. Brett, R. B. Appleby, R. De Maria, J. Barranco Garcia, R. Tomás Garcia, B. Hall, and G. Burt, Accurate crab cavity modeling for the high luminosity Large Hadron Collider, Phys. Rev. ST Accel. Beams 17, 104001 (2014).

[21] D. R. Brett, R. B. Appleby, G. Burt, and B. Hall, Particle trajectories in a four rod crab cavity, Nucl. Instrum. Methods Phys. Res., Sect. A 734, 79 (2014).

[22] R. Calaga, R. De Maria, J. Barranco Garcia, M. Giovannozzi, A. Grudiev et al., Study of multipolar RF kicks from the main deflecting mode in compact crab cavities for LHC, in Proceedings of the 3rd International Particle Accelerator Conference, New Orleans, LA, 2012 (IEEE, Piscataway, NJ, 2012), pp. 1873-1875.

[23] J. Barranco Garcia, R. De Maria, A. Grudiev, R. Tomás Garcia, R. B. Appleby, and D. R. Brett, Long term dynamics of the high luminosity Large Hadron Collider with crab cavities, Phys. Rev. Accel. Beams 19, 101003 (2016).

[24] SM-18 Test Facilities, CERN, https://espace.cern.ch/tedep-msc-tf-archived2018/SitePages/Home.aspx.

[25] J. Delayen, Self-Excited Loop, LLRF workshop (Jefferson Lab, Newport News, Virginia, 2001).

[26] S. Verdu-Andres, SPS DQWCC tuning, Appendix C, https://edms.cern.ch/document/1747736/2 (2018).

[27] Methodical Accelerator Design, http://madx.web.cern.ch/ $\operatorname{madx} /$.

[28] F. Schmidt, SixTrack Version 4.2.16 single particle tracking code treating transverse motion with synchrotron oscillations in a symplectic manner, CERN Report No. CERN/SL/ 9456, 2012, http://sixtrack.web.cern.ch/SixTrack/.

[29] R. De Maria, A. Mereghetti, and K. Sjobak, SixTrack project: status, runtime environment, and new developments, 13th International Computational Accelerator Physics Conference, Key West, Florida, USA (2018), https://doi .org/10.18429/JACoW-ICAP2018-TUPAF02.

[30] K. Sjobak, R. De Maria, E. McIntosh, A. Mereghetti, J. Barranco Garcia, M. Fitterer, V. Gupta, and J. Molson, New features of the 2017 SixTrack release, IPAC'17 conference proceedings, Copenhagen, Denmark (2017), pp. 3815-3818, http://inspirehep.net/record/1626386/files/ thpab047.pdf.

[31] G. Robert-Demolaize, R. Assmann, S. Redaelli, and F. Schmidt, A new version of SixTrack with collimation and aperture interfaces, in Proceedings of the 21st Particle 
Accelerator Conference, Knoxville, TN, 2005 (IEEE, Piscataway, NJ, 2005), pp. 4084-4086.

[32] D. Mirarchi, A. Bertarelli, R. Bruce, F. Cerutti, P. Hermes, A. Lechner, A. Mereghetti, E. Quaranta, S. Redaelli, H. Garcia Morales, R. Kwee-Hinzmann, and R. B. Appleby, Cleaning performance of the collimation system of the high luminosity Large Hadron Collider, IPAC'16 conference proceedings, Busan, Korea (2016), pp. 2494-2497, https:// cds.cern.ch/record/2207409/files/wepmw030.pdf.

[33] A. Santamaría García, Experiment and machine protection from fast losses caused by crab cavities in the high luminosity LHC, Ph.D. thesis, EPFL, 2018.

[34] R. Bruce et al., Simulations and measurements of beam loss patterns at the CERN Large Hadron Collider, Phys. Rev. ST Accel. Beams 17, 081004 (2014).

[35] K. Sjobak, H. Burkhardt, R. De Maria, A. Mereghetti, and A. Santamaría García, General functionality for turn-dependent element properties in SixTrack, IPAC'15 conference proceedings, Richmond, Virginia, USA (2015), pp. 468-471.

[36] K. Sjobak, V. K. Berglyd Olsen, R. De Maria, M. Fitterer, A. Santamaría García, H. Garcia-Morales, A. Mereghetti, J. F. Wagner, and S. J. Wretborn, Dynamic simulations in SixTrack, in Proceedings of ICFA Mini-Workshop on Tracking for Collimation in Particle Accelerators, CERN, Geneva, Switzerland, 2015; CERN Yellow Reports: Conference Proceedings, Vol. 2/2018, CERN-2018-011CP (CERN, Geneva, 2018).
[37] B. Yee-Rendon, R. Lopez-Fernandez, J. Barranco, R. Calaga, A. Marsili, R. Tomás, F. Zimmermann, and F. Bouly, Simulations of fast crab cavity failures in the high luminosity Large Hadron Collider, Phys. Rev. ST Accel. Beams 17, 051001 (2014).

[38] K. Hosoyama et al., Development of the KEK-B superconducting crab cavity, in Proceedings of the 11th European Particle Accelerator Conference, Genoa, 2008 (EPS-AG, Genoa, Italy, 2008), pp. 2927-2931.

[39] I. Bejar Alonso and L. Rossi, HiLumi LHC technical design report: Deliverable: D1.10, CERN Report No. CERNACC-2015-0140, 2015 https://cds.cern.ch/record/2069130.

[40] K. Sjobak and A. Santamaría García, Crab cavity failure modes and mitigation, 6th HL-LHC collaboration meeting, Paris, France, https://indico.cern.ch/event/549979/ contributions/2295073/attachments/1371504/2080340/ 2016-11-14_HLLHC_Paris-CrabFailures-Kyrre.pdf (2016).

[41] O. Brüning, P. Collier, P. Lebrun, S. Myers, R. Ostojic, J. Poole, and P. Proudlock, LHC Design Report CERN Yellow Reports: Monographs (CERN, Geneva, 2004), https://cds.cern.ch/record/782076.

[42] R. Calaga, L. Ficcadenti, E. Metral, R. Tomás, J. Tuckmantel, and F. Zimmermann, Proton-beam emittance growth in SPS coasts, in Proceedings of the 3rd International Particle Accelerator Conference, New Orleans, LA, 2012 (IEEE, Piscataway, NJ, 2012), pp. 3737-3739. 